\title{
IMPALAS: Investigation of MagnetoPause Activity using Longitudinally-Aligned Satellites - a Mission Concept proposed for the ESA M3 2020/2022 Launch.
}

Christopher J. Owen, Olaf Amm, Roberto Bruno, Johan De Keyser, Malcolm Dunlop, Jonathan Eastwood, Andrew N. Fazakerley, Dominique Fontaine, Colin Forsyth, Hiroshi

Hasegawa, Petr Hellinger, David Hercik, Christian Jacquey, Steven Milan, Joachim Raeder, David G. Sibeck, Stepan Stverak, Pavel Travnicek, Andrew P. Walsh, James Wild.

Corresponding Author: Prof. Christopher J. Owen

Address:

Mullard Space Science Laboratory, University College London, Holmbury St. Mary, Dorking, Surrey, RH5 6NT, United Kingdom

E-mail:

Telephone:

cjo@mssl.ucl.ac.uk

Fax:

$+44-1483-204281$

$+44-1483-278312$

Affiliations:

C.J. Owen, A.N. Fazakerley, C. Forsyth, A.P. Walsh, Mullard Space Science Laboratory, University College London, Holmbury St. Mary, Dorking, Surrey, RH5 6NT, UK;

O. Amm

Finnish Meteorological Institute, Finland;

R. Bruno INAF-IFSI, Italy;

J. De Keyser

Institute for Space Aeronomy, Belgium;

M. Dunlop

Rutherford Appleton Laboratory, UK;

J. Eastwood Imperial College, UK;

D. Fontaine

Laboratoire de Physique des Plasmas, Ecole Polytechnique, France;

H. Hasegawa Institute of Space and Astronautical Science (ISAS), Japan;

P. Hellinger, D. Hercik, S. Stverak, P. Travnicek, Academy of Sciences of the Czech Republic;

P.Travnicek also at University of California at Berkeley, USA;

C. Jacquey

Centre d'Etude Spatiale des Rayonnements (CESR), France;

S. Milan

University of Leicester, UK;

J. Raeder University of New Hampshire, USA;

D. Sibeck

NASA/Goddard Space Flight Center, USA;

J. Wild University of Lancaster, UK. 


\section{Abstract}

The dayside magnetopause is the primary site of energy transfer from the solar wind into the magnetosphere, and modulates the activity observed within the magnetosphere itself. Specific plasma processes operating on the magnetopause include magnetic reconnection, generation of boundary waves, propagation of pressure-pulse induced deformations of the boundary, formation of boundary layers and generation of Alfven waves and field-aligned current systems connecting the boundary to the inner magnetosphere and ionosphere. However, many of the details of these processes are not fully understood. For example, magnetic reconnection occurs sporadically, producing flux transfer events, but how and where these arise, and their importance to the global dynamics of the magnetospheric system remain unresolved. Many of these phenomena involve propagation across the magnetopause surface. Measurements at widely-spaced $\left(\Delta \sim 5 R_{E}\right)$ intervals along the direction of dayside terrestrial field lines at the magnetopause would be decisive in resolving these issues. We describe a mission carrying a fields and plasmas payload (including magnetometer, ion and electron spectrometer and energetic particle telescopes) on 3 identical spacecraft in synchronized orbits. These provide the needed separations, with each spacecraft skimming the dayside magnetopause and continuously sampling this boundary for many hours. The orbits are phased such that (i) all 3 spacecraft maintain common longitude and thus sample along the same magnetopause field line; (ii) the 3 spacecraft reach local midday when northern European ground-based facilities also lie near local midday, enabling simultaneous sampling of magnetopause field lines and their footprints.

Key Words: Magnetopause, Magnetic Reconnection, Solar Wind - Magnetosphere Coupling, Cosmic Vision.

\section{Introduction}

In July 2010 the European Space Agency issued a call for proposals from the scientific community for candidate concepts for a medium-size (M-class) mission, the third launch opportunity for this class of mission (M3) under ESA's Cosmic Vision 2015-2025 program. This call came against a background in which the European space plasmas community had previously responded to the earlier M1/M2 opportunity with a proposal for a multispacecraft mission entitled 'Cross-Scale' (Schwartz et al., 2008). This mission concept, which had broad science goals addressing fundamental processes relevant to space and astrophysical plasma systems, underwent a full assessment study phase (Schwartz et al., 2009), but was not ultimately selected for the M1/M2 launch opportunity.

Lessons from the Cross-Scale Assessment Study include an understanding that the ESA M-Class budget, fixed at 470 MEuros for the M3 opportunity, is sufficient for only 2 or 3 ESA-sponsored spacecraft capable of making science-quality in situ fields and plasma measurements. A further lesson from the Cross-Scale experience is that addressing very broad science goals results in a susceptibility to mission resource creep, and difficulty in maintaining full community support when descopes become necessary to keep the mission concept within resource envelopes.

In contrast, the NASA/THEMIS mission [Angelopoulous, 2008] shows that significant progress on major unanswered questions in magnetospheric physics can be made by tailoring a multi-spacecraft mission to make specific measurements in specific locations in the magnetosphere, and that this progress will be augmented if ground-based capability is included as an extra measurement point. 
ESA's Cluster mission has addressed, and NASA's MMS mission will address, aspects of magnetopause dynamics, but on scales that are small relative to the tangential extent of this boundary. However, magnetopause phenomena often involve propagation of waves and other structures across this surface. In many cases, this motion is strongly influenced by the northward pointing direction of the terrestrial magnetic field. Understanding of these phenomena could be enhanced by measurements made at more widely-spaced $(\Delta s \sim 5$ $R_{E}$ ) points along the direction of dayside terrestrial magnetic field at the magnetopause.

The issues noted above suggest that a mission proposal with a set of focussed science goals, primarily targeting the dayside magnetopause, and an equivalently focussed payload is a sound concept for an ESA M-Class launch opportunity. Under this philosophy, therefore, we describe in this paper a 3-spacecraft mission which we have called IMPALAS (Investigation of MagnetoPause Activity using Longitudinally-Aligned Satellites). We demonstrate in the next section that there are compelling science goals to be met, while in section 3 we detail the mission profile needed to achieve the objectives. Section 4 describes the model payload for the mission and section 5 the key parameters for the spacecraft bus. Finally, section 6 summarises possible technology developments, some of the expected programmatic issues and the current status of the mission.

2 Scientific Objectives and Requirements

\subsection{Science Rationale: Why target Earth's magnetopause?}

The magnetopause is the boundary between the solar and terrestrial plasma regimes. It is a critical interface in the field of solar-terrestrial relations, in that the coupling processes that ultimately control all magnetospheric dynamics occur there. These include some fundamental plasma processes, such as magnetic reconnection, particle acceleration and boundary wave generation. In the regions surrounding this interface other important processes, such as plasma turbulence, the creation of polarization electric fields due to finite gyroradius effects, and wave-particle interactions, can also be found. The magnetopause is the key interface for defining the influence of 'space weather' on the Earth system, with the effects of, for example, Solar Particle Events (SPEs) and Coronal Mass Ejections (CMEs) mitigated by, or transmitted through, this boundary before they affect near-Earth space. The magnetopause is arguably the most readily accessible analogue to other space and astrophysical plasma boundaries. There is much interest in the magnetopause at the other planets. Generally speaking, telemetry constraints on missions to the other planets mean this boundary is always more poorly resolved and sampled than at the Earth, such that knowledge of the Earth system is crucial to put the more distant observations in context. Plasma boundaries in the solar wind, such as the heliospheric current sheet, are hard to study in detail as they rapidly pass the spacecraft at the high solar wind speeds. Other plasma boundaries, for example the heliopause (the boundary between solar and interstellar plasma regimes), or those between other stellar and galactic spheres of influence, cannot be directly sampled. Thus understanding the interactions that occur at the terrestrial magnetopause can provide important ground truth for understanding these more remote interaction regions.

Over the last few decades a significant number of spacecraft encounters with the magnetopause have occurred. Early observations consist of many brief single spacecraft traversals of the magnetopause, which generally occur when the boundary sweeps past the spacecraft as it rapidly moves in and out in response to changes in the solar wind ram pressure. The ESA/Cluster mission has recently made multi-point measurements over relatively small scales at the magnetopause. This provides insights into the underlying 
physics of the interactions, for example revealing the detailed fields and currents in the vicinity of active reconnection regions. Further progress in understanding such plasma microphysics can be expected from the NASA Magnetospheric Multi-Scale Mission (MMS). This 4 spacecraft mission (launch 2014) will make measurements of the magnetosphere, including the magnetopause, but at much smaller separations than Cluster. Conversely, we have only a few rare and fortuitous spacecraft conjunctions over larger scales. Most recently, for example, the Cluster and Double Star missions provided a handful of events in which 2 spacecraft sample the magnetopause nearly simultaneously, but at large separations. Nevertheless, these sporadic observations are extremely useful in providing indications of the more global dynamics of the magnetopause, for example in tracking the motion of boundary disturbances.

Significant progress could thus be made in understanding the global dynamics of the magnetopause, if we could generate a statistically significant number of 'controlled' conjunctions with multiple spacecraft taking simultaneous in situ measurements at the magnetopause and spread relatively widely compared to the Cluster mission ( $\left.\Delta s \sim 5 R_{E}\right)$. This would be particularly so if that separation were along, say, a reconnecting magnetic field line. The value of these in situ measurements could be considerably enhanced if they were also made in association with concurrent measurements of the ionosphere at or near the foot-points of the terrestrial magnetic field lines that lie just inside the dayside magnetopause. Such complementary measurements could be made by remote sensing, from the spacecraft, of the auroral emissions around these foot-points and/or by designing a mission which has magnetic conjunctions between the spacecraft and ground-based facilities making observations in the vicinity of these foot-point regions. Some examples of top-level science goals that could be addressed by such measurements are summarised in Table 1 and described in the remainder of this section.

\subsection{Steady State Reconnection at the Dayside Magnetopause}

Magnetic Reconnection is a fundamental and ubiquitous process within plasma systems throughout the universe. It breaks down the barriers between neighbouring plasmas, releasing energy from their magnetic fields, transferring material and momentum between those plasmas, and accelerating a part of the plasma population to high energies. Astrophysical plasma systems in which reconnection is expected to play a significant role in their dynamic evolution include the Sun and other stars and planetary systems at all stages of their life cycles.

The global dynamics of the Earth's magnetosphere are dominated primarily by the action of magnetic reconnection at the dayside magnetopause, as first recognised by Dungey (1961). When a strong magnetic shear exists across this dayside boundary, primarily during periods when the highly-variable IMF direction is significantly different to that of the terrestrial field, magnetic reconnection may occur, either locally or on a semi-global scale. This results in a coupling between the solar wind and the magnetosphere, which in turn results in a 'peeling' away of magnetic flux tubes from the dayside magnetopause surface, an acceleration of particles along field lines in a direction away from the reconnection site, a transportation of magnetic flux and particles over the poles, the generation of fieldaligned currents connecting the outer magnetosphere to the ionosphere, and an eventual storage of that flux in the nightside magnetospheric tail. The ongoing flux storage eventually destabilises the nightside tail region of the terrestrial magnetosphere, leading to a 'magnetic substorm', which results in a major reconfiguration of the magnetosphere and disruptions extending down into the polar ionospheric regions. Long periods of enhanced coupling between the solar wind and the magnetosphere result in magnetic storms. Thus magnetic reconnection at the dayside magnetopause has the key controlling influence on 
'space weather' effects within the terrestrial environment which can have serious consequences for our ground- and space-based technological assets.

Despite having developed a workable understanding of the consequences of magnetic reconnection on the dayside magnetopause, there remain open a large number of significant scientific questions about the process itself. Key questions include:

- Where does reconnection occur on the magnetopause for given solar wind and interplanetary magnetic field conditions?

- Does reconnection occur along single or multiple extended reconnection lines or in patches?

- Does reconnection occur steadily or in transient bursts?

- What governs the transition between steady and bursty, patchy and extended, equatorial and high-latitude reconnection?

Multipoint case and statistical studies of the locations and characteristics of magnetopause reconnection as a function of solar wind/magnetosheath conditions are essential to distinguish between competing models for the reconnection process. While most models invoke subsolar reconnection for southward IMF orientations, some predict that reconnection remains subsolar for northward IMF conditions, others that it shifts to higher latitudes, and still others that it continues at both low and high latitude (e.g. Trattner et al., 2007 and references therein). In the absence of appropriate spacecraft separations, we do not know whether reconnection occurs along an extended neutral line stretching several or many Earth radii (RE) across the magnetopause, in small singular or multiple patches, or whether indeed it occurs in a quasi-steady manner at all.

One of the prime goals of the IMPALAS mission should be to determine the location of the MP reconnection site(s) for given IMF conditions. Signatures of reconnection include relatively high-speed flows of plasma particles along field lines adjacent to the dayside magnetopause, which often show a characteristic dispersion in energy and a distinct low energy cut-off due to the sling-shot action of the reconnected magnetic field. The configuration of the mission should allow the return of a large number of simultaneous measurements of such particle dispersions/cut-offs at multiple positions in the magnetopause boundary layer broadly occupied by the same reconnected field lines. Very few such cases exist in current databases, since the required conjunctions between spacecraft have to date occurred only fortuitously and very rarely. The IMPALAS concept should make these a routine occurrence, by placing multiple spacecraft in permanent conjunction in their orbits. Once the data have been generated, the methodology applied to a few case studies by Fuselier et al. (2005), and illustrated in Figure 1, could be used to determine the relative position of the reconnection site to the spacecraft on every conjunction event. We could then determine how that location changes with variations in the IMF by comparison with observations of the prevailing solar wind conditions, establish whether the configuration of the magnetic field on either side is anti-parallel or not, whether this depends on the external conditions, and determine the temporal evolution of the reconnection site over timescales of minutes to hours. Finally, conjugate ionospheric measurements, such as HF radar observations of the ionospheric flows excited by the motion of the foot-points of the reconnected field lines (e.g. Chisham et al., 2000) being sampled by the space-segment, the longitudinal extent of the processes observed in space could be inferred. 


\subsection{Transient Reconnection at the Dayside Magnetopause}

It has long been recognised that magnetic reconnection may operate in a transient and/or sporadic and patchy manner on the dayside magnetopause. Russell and Elphic (1978, 1979) introduced the concept of the "Flux Transfer Event (FTE)" to interpret characteristic magnetic field perturbations observed by ISEE-1 and -2 in the vicinity of the low-latitude magnetopause. The plasma observed within FTE's often consists of a mixture of magnetospheric and magnetosheath plasma (e.g. Thomsen et al., 1987) usually associated with fast plasma flows (Paschmann et al., 1982) and ion D-shaped distributions (Smith and Owen, 1992). Moreover, most FTE's are observed during southward IMF conditions (e.g. Rijnbeek et al., 1984). These observations are strong indications that these structures are isolated open flux tubes most likely generated with transient and localised magnetic reconnection occurring on the nearby magnetopause.

Again, despite this general understanding of the origin and nature of these events, there remain many unanswered scientific questions concerning their formation, structure and evolution, including:

- What are the preferred locations for FTE formation under different solar wind and IMF conditions?

- Do FTEs form between pairs of reconnection lines or at single reconnection lines. Do they form in pairs or as isolated single events?

- What are the spatial extent and shapes of FTE's? How does the structure of FTEs vary as a function of location along the magnetopause?

- How do FTE's move and evolve across the dayside magnetopause?

- Do FTE's contribute significantly to the global open flux cycle?

- Do FTE's form only in the winter hemisphere, as recent simulations (Raeder et al. 2006) suggest?

- What is the ultimate fate of FTE's - How far do they travel from the point of origin?

- How do FTE's at the magnetopause affect the polar cusps and polar ionosphere?

As for steady-state reconnection, case and statistical surveys of FTEs employing appropriately-spaced spacecraft would prove decisive in describing the formation and evolution of FTE's on the magnetopause and determining their significance. This would allow us to validate both analytical and numerical simulation models for their motion across the dayside magnetopause (e.g. Cooling et al., 2001), and thereby determine, on a regular basis, the location of their formation as a function of the prevailing conditions upstream of the magnetopause. In addition, obtaining observations of matched pairs of FTE's in both the southern and northern hemispheres will not only help this process through the ability to triangulate back to a common point of origin, but will help answer some fundamental questions as to the role of FTE's in the global magnetic flux cycle. For example, recent simulations by Raeder (2006) have suggested that FTE's do not occur in pairs, as the original interpretations of their formation require, but form only in the winter hemisphere through re-reconnection of already opened field lines, as illustrated in Figure 2. Simultaneous measurements on the magnetopause in both north and south hemisphere, as would be available from the IMPALAS mission, would provide a definitive answer to this open question. Moreover, Milan et al. (2004) noted the apparent discrepancy in the typical size of an FTE, in terms of its magnetic flux content, determined from spacecraft observations and the global rate of flux transport derived from ionospheric radar measurements. This implies FTE's occur on the magnetopause at a significantly higher rate than has been observed. This may be a result of the mostly relatively short 'dwell' times of previous missions in positions close enough to the relevant parts of the 
magnetopause, which means that most FTE's may be missed. The IMPALAS mission concept aims to maximise this 'dwell' time and thereby obtain the observations necessary to make a definitive assessment of the rate that open flux is added to the magnetosphere by FTE's.

\subsection{Magnetopause Boundary Waves and Deformations}

As well as the reconnection phenomena that are intrinsic to the magnetopause current sheet, this boundary is also susceptible to local deformations which create waves travelling across the surface. Some of these are externally driven, for example by changes in pressure in the upstream solar wind. Others may be generated by instabilities intrinsic to the magnetopause, which may also cause a local magnetopause deformation. Due to the flow shear that generally exists across the magnetopause boundary between the dense, fast-flowing magnetosheath and the more tenuous, but more static plasma of the magnetosphere proper, the Kelvin-Helmholtz $(\mathrm{KH})$ instability is often invoked as a source of magnetopause boundary motion (e.g. Hasegawa et al., 2009, see Figure 3). Other processes intrinsic to the magnetopause also generate boundary waves. For example, Owen et al. (2008) recently interpreted Cluster observations in the wake of an FTE as the passage of a 'travelling magnetopause erosion event', in which the indentation left by the removal of magnetic flux from the dayside magneto-sphere by an FTE was driven across the magnetopause surface by the action of the magnetosheath flow. This interpretation has since been confirmed by simulation (Kuznetsova et al., 2009). While surface deformations are carried tailward because of magnetosheath flow, their propagation along magnetic field lines has been studied much less. The IMPALAS configuration would be very well suited to this purpose. Specific open questions that could be addressed with observations from the IMPALAS mission include:

- How do solar wind pressure pulses deform the magnetopause, and how does that deformation move and evolve across the magnetopause surface?

- Where (and when) do KH and other boundary waves develop on the magnetopause, and how is this affected by the upstream solar wind conditions?

- What is the role of the $\mathrm{KH}$ instability in the transport processes operating at the terrestrial magnetopause, particularly under northward IMF conditions?

- How do the indentations resulting from magnetic flux erosion dissipate across the magnetopause?

- Can solar wind pressure pulses generate FTE-like signatures? If so, how do the magnetosheath signatures differ from the magnetospheric signatures?

\subsection{Magnetopause Boundary Layers}

Previous missions carrying in situ plasma packages have revealed that a variable set of boundary layers can generally be found on either side of the magnetopause current layer, with their occurrence and location being controlled by the upstream solar wind and IMF conditions.

Under certain conditions a region forms upstream of the magnetopause in which the magnetic field piles up ahead of the boundary, and the plasma is 'squeezed out' away from the region along the field direction. However, observations of this plasma depletion layer (PDL) are sporadic, indicating that its formation, thickness, extent and degree of plasma depletion are highly variable, and most likely depend heavily on the prevailing solar wind and magnetopause conditions. In addition, the inherent flow pattern in the solar wind and magnetosheath (e.g. laminar or turbulent flows in the latter) may affect the nature, size and location of the PDL regions. One particularly relevant point is the degree to which 
this boundary may extend to regions of high-latitude and particularly to cover regions of the magnetopause poleward of the cusp. During periods when the PDL is absent, these regions are expected to be adjacent to magnetosheath flows which are super-Alfvènic, a condition which will limit the occurrence of magnetic reconnection in this region (Cowley and Owen, 1989). However, if a PDL extends to high latitude, this may reduce the flows in this region, and/or increase the local Alfvèn speed, such that the flow is sub-Alfvènic and susceptible to quasi-steady reconnection processes. This in turn has important consequences for the structure and dynamics of the magnetosphere under northward IMF conditions.

Immediately downstream from the magnetopause boundary, a further set of boundary layers is known to exist. The plasma in these layers generally consists of a mixture of magnetosheath and magnetospheric plasma. At high-latitudes, a natural explanation for such a boundary layer (the high-latitude boundary layer, HLBL) is the mixing and acceleration of plasmas as a consequence of the magnetic reconnection process. The plasmas in such layers have been observed to have the characteristics imposed by reconnection, such as low-velocity cut-offs in the distribution (e.g. Smith and Rodgers, 1991) and velocity-dispersed layers (e.g. Gosling et al., 1990). A second class of boundary layer has also been identified and given the term low-latitude boundary layer (LLBL), although it may encompass layers formed by a number of different processes which have yet to be unambiguously identified.

The global nature of these boundary layers and the processes leading to their formation at different locations and under different upstream conditions is still not fully understood. Specific science questions which remain open, but would be readily addressed with observations from the IMPALAS mission, include:

- Where do the boundary layers (LLBL and HLBL) form under different solar wind conditions?

- What is the thickness and magnetic topology of the LLBL as a function of distance along the field direction from the subsolar point?

- What are the relative roles of reconnection and diffusive entry (e.g. breaking of $\mathrm{KH}$ vortices) in the generation of the LLBL?

- What plasma waves are generated in the magnetopause? How does the intensity of such waves affect diffusive plasma transfer across the magnetopause to form the boundary layers?

- What are the timescales for the generation/dissipation of the HLBL and LLBL following a change in solar wind conditions?

- How do these boundary layers map to the ionosphere?

- Under what solar wind conditions does a plasma depletion layer arise at different locations on the magnetopause?

- What is the PDL location, thickness, depth of depletion, etc. as a function of distance from the subsolar point?

- How far poleward does the PDL extend along the MP - does it support steady-state reconnection occurring poleward of the cusps?

\subsection{The Impact of Solar Wind Transients on the Magnetosheath and Magnetopause}

The magnetosheath is the global boundary layer occupying the region between the bow shock upstream and the magnetopause downstream. It contains solar wind plasma which 
has been shocked, heated and deflected in order to pass around the magnetospheric cavity. Observations of this region have shown that the flow pattern can at times appear very turbulent and at others more laminar in nature. Other observations are consistent with the occurrence of mirror mode waves convecting with the magnetosheath flow (e.g. Horbury and Lucek, 2009). The nature of the flow, particularly immediately upstream of the magnetopause (for example, whether the flow pattern forms a singular stagnation point or a stagnation line (Phan et al. 1994), or whether there are asymmetries in the flow patterns from north to south (similar to those reported for dawn-dusk by Paularena et al., 2001)) is a critical input to our models of magnetospheric dynamics.

Moreover, the effects of disturbances and discontinuities inherent to the upstream solar wind flow on the magnetosheath and magnetopause system are also critical to magnetospheric dynamics. For example, Sibeck et al. (1999) demonstrated that under certain circumstances when the IMF is quasi-radial, a hot flow anomaly (HFA) can develop upstream of the bow shock which can have a significant effect on the pressure profile being transmitted through the magnetosheath and ultimately to the magnetopause. Sibeck et al., (2004) argued that pressure reductions associated with HFA's can cause a very significant outward deformation of a relatively localised region of the magnetopause surface, although we do not currently have measurements which can definitively confirm this interpretation, nor determine the extent of such a region, how it moves across the dayside magnetopause or how it evolves as it does so. The propagation of a number of other transients in the solar wind, such as interplanetary shocks and current sheets, through the magnetosheath and along the magnetopause surface remain similarly poorly understood. For example, Sibeck (1990) has argued that transient solar wind pressure pulses produce ripples on the magnetopause surface which mimic many of the signatures of FTE's. Hence some of the key questions that still need to be addressed concerning the structure of the magnetosheath and the effects of transients include:

- How does the global structure of the magnetosheath vary as a function of IMF and solar wind parameters?

- Does the global structure of the magnetosheath exhibit north-south and/or dawn-dusk east-west asymmetries?

- Does the overall magnetosheath flow structure vary significantly for different conditions (does the magnetosheath exhibit turbulent or laminar flows, are there stagnation lines or points in the flow structure)?

- How do disturbances, discontinuities and waves propagate within the magnetosheath and how and where can they impact the magnetopause?

- How can we reliably separate signatures of transient pressure pulses from those of FTE's at the magnetopause?

\subsection{Propagation of Magnetopause Phenomena to the Polar lonosphere}

Many of the phenomena described above are directly coupled along terrestrial magnetic field lines into the auroral ionosphere. This latter region can be well studied using existing comprehensive networks of ground magnetometers, HF radars, all-sky cameras, incoherent radars, ionosondes, etc., particularly in the Scandanavian and Canadian local time sectors. The IMPALAS mission concept, which seeks to make space-based observation in magnetic conjunction with such networks, therefore offers an opportunity to further our understanding of the dynamics of the coupled magnetosphere-ionosphere system driven by momentum and energy transfer from the solar wind at the dayside magnetopause. For example, the production of open magnetic flux and its transport into 
the tail, will drive ionospheric flows poleward and into the polar cap from the dayside auroral region. The global configuration of these auroral zone and polar cap flows can be determined using the multi-point measurements available from the SuperDARN network of ionospheric radars, while the currents driven in the ionosphere as a result of this coupling can be determined using measurements from appropriately located networks of ground magnetometer stations. From these data, it is possible to determine, for example, the position of the ionospheric boundary between open and closed field lines, which maps to the edge of the HLBL that would be regularly sampled at multiple points by the IMPALAS spacecraft. Moreover, these measurements can be used to determine the longitudinal extent of the active reconnection site, and the overall production rate of open magnetic flux (Chisham et al., 2004), which cannot be determined from space-based measurements alone. With magnetically conjugate measurements from multiple points along reconnected field lines, we will be able to determine the electromotive force delivered towards the ionosphere, and the outgoing reflected part (Amm et al., 2010). This would enable us to determine the contribution of the reflection and polarization processes to the energy deposition in the ionosphere, and to determine the parts of the horizontal ionospheric current systems related to these processes. This approach will also allow us to resolve the conundrum of the apparent imbalance between open flux transported in individual FTE's observed at the magnetopause, and that determined from ground-based measurements (Milan et al., 2003). Finally, a combined IMPALAS-ground based study of the mapping of boundary waves and deformations from the magnetopause to the ionosphere will help reveal the origin and coupling of ULF wave power into the inner magnetosphere and ionosphere. In summary, some specific questions that could be addressed by a combined IMPALAS-ground-based campaign include:

- How do processes (reconnection, FTE's, boundary waves) occurring on the day-side magnetopause map down the field lines and affect the auroral ionosphere?

- How does the ionosphere provide feed-back to processes occurring on the day-side magnetopause - can this saturate the reconnection rate, for example?

- How does the connection to the ionosphere affect KH stability of the boundary and the development of the $\mathrm{KH}$ and other boundary waves?

- What is the width of the merging gap (the footprint of reconnection) in the ionosphere as a function of magnetopause and IMF conditions?

- What causes inter-hemispherical asymmetries in the auroral forms and polar ionospheric flow patterns?

- What precipitation patterns and optical features do events at the magnetopause generate?

\subsection{Other Magnetospheric Science Topics that may be Serendipitously Addressed}

The IMPALAS concept orbit design described below is tuned to answer the magnetopause-related science questions posed above. However, orbital dynamics means the 3 spacecraft must visit other parts of the magnetosphere at varying separations over the course of a year. For example, the spacecraft will also pass through the transition region between dipolar and stretched tail-like magnetic field configurations in the nightside magnetotail, a key region for the onset of magnetospheric substorm dynamics. The IMPALAS configuration will thus provide new insight into cross-tail current disruption for example by identifying possible current density thresholds. Furthermore, the auroral imagers on the IMPALAS spacecraft will also be able to observe any conjugacies (or lack of thereof) in the substorm aurora in opposite hemispheres, and link these to the particle and field characteristics observed in situ by each spacecraft. This potentially provides a 
key link between processes occurring in the onset region in the magnetotail and those occurring in the conjugate auroral ionosphere, and thereby could provide important tests of models of substorm onset.

We note that the separation of the 3 IMPALAS spacecraft changes over a single orbit, so there are opportunities to investigate the magnetosphere and the physical processes operating within it at a variety of latitudinal spacecraft separations. At the opposite extreme to the separation used for the prime science discussed above, the spacecraft will all be relatively close as they cross the ecliptic plane. At this point in the orbit, the spacecraft are likely to be almost radially aligned with a separation of the order of $2 R_{E}$. This configuration also has the potential to return highly valuable observations pertaining to substorm dynamics. For example, the radial profile, formation and evolution of energetic particle injection fronts, dipolarisation fronts, bursty bulk flows and the region of flow braking and current disruption (e.g. Nakamura et al., 2009, Spanswick et al., 2010) could be determined by spacecraft in this configuration.

\subsection{IMPALAS Measurement Requirements}

The IMPALAS mission primarily targets the dynamics of the dayside magnetopause over relatively large scales compared to previous missions. The primary requirement is to make in situ magnetic field and plasma measurements over long durations at 3 points spaced at several $R_{E}$ along the terrestrial magnetic field line direction just inside the average magnetopause position. The regions targeted in the science objectives above will then be sampled regularly due to the natural variability in the actual position of the magnetopause. Given the typical time and spatial scales of the phenomena being targeted, the in situ instruments should return measurements in the $1-10$ second range. The low telemetry demands of the fields instruments means data rates for these instruments could be 1 second cadence. However, the necessity of the particle instruments to use spacecraft spin to sample the full sky, implies a 3-6 second cadence for full 3D measurements. In addition, measurements of the dynamics of the auroral footpoint of the field line will significantly add to the science return from the mission. The IMPALAS mission should therefore be capable of imaging the dayside auroral zone, ideally in both hemispheres. The measurements required to meet the science goals described above, the instruments that therefore need to be included in the payload, and a brief description of their role in meeting the science goals are listed in Table 2.

\subsection{Other Measurement Requirements}

The IMPALAS mission should make longitudinally conjugate measurements, which in principle provide multiple measurements along the same field line when the spacecraft are located at and just inside the dayside magnetopause. However, if the orbits are phased appropriately, a number of other key measurements can be made when the spacecraft are at maximum separation and also in magnetic conjunction with relevant ground-based facilities, such as the ground-based magnetometer arrays of the IMAGE network (or similar networks in the Canadian sector). IMAGE consists of 31 magnetometer stations in Scandinavia which enable the study of auroral electrojets and moving two-dimensional current systems above this region with $\sim 50 \mathrm{~km}$ resolution. These observations are complemented by, for example, high time-resolution measurements ( 2 mins) of the ionospheric flow vectors in the same sector by the CUTLASS radar system and measurements by the international EISCAT radar facility and the EISCAT Svalbard radar. The CUTLASS radars are part of the wider SuperDARN radar network, combined measurements from which can provide the ionospheric flow pattern over the entire auroral and polar cap region, thereby providing a global context for the IMPALAS in situ measurements. 


\section{Mission Profile}

\subsection{Overview}

The mission assembled to completely address the science goals described above needs to provide simultaneous observations of the terrestrial dayside magnetopause and/or its environs at a minimum of 3 points with latitudinal separation (i.e. along the direction of a terrestrial magnetic field line) of order $5 \mathrm{R}_{\mathrm{E}}$. Variations of this distance are likely to be scientifically valuable to the outcome of the mission and are therefore highly desirable. The mission should thus consist of a baseline of 3 spacecraft, which for the purposes of the discussion here we designate 11,12 and 13 .

\subsection{Orbit requirements}

The overall requirement for the selected orbits is to provide extended periods of conjunction of the 3 spacecraft widely separated in latitude along the magnetic field (longitudinal) direction at the known average location of the dayside magnetopause. The latitudinal separation requires that the 3 spacecraft have individual orbits in 3 separate planes. The requirement for longitudinal conjunction suggests that the 3 orbits should have exactly the same period, or have enough fuel to correct for significant medium term drifts. The requirement for conjunctions at the dayside magnetopause implies that each of the orbits be chosen to minimise the average net distance from the known average location of the dayside magnetopause in the 9 to 15 hours magnetic local time sector. Furthermore, it is highly desirable that the orbits are phased so that the main science periods, when the spacecraft are widely separated at local noon, coincide with relevant European ground-based facilities also being located at local noon.

A possible example of the kind of orbit envisioned for the IMPALAS mission is shown in Figure 4. I1 could be placed in a circular orbit with a $10.65 R_{E}$ radius $\left(1 R_{E}=1\right.$ Earth Radius $=6371 \mathrm{~km}$ ) at $0^{\circ}$ inclination. 12 should be placed in a slightly eccentric orbit with apogee $\sim 11 \mathrm{R}_{\mathrm{E}}$ and $+30^{\circ}$ inclination. Finally $\mathrm{I} 3$ should be placed in a similarly eccentric orbit as 12 but with $-30^{\circ}$ inclination. The point of these orbits is that each spacecraft should then have an exactly 2 day period which 'skims' dawn-to-dusk very close to the average position of the dayside magnetopause, as defined, for example, in the Fairfield (1971) model. Each of the 3 orbits can thus be phased so that each spacecraft remains in close longitudinal (magnetic) alignment with the other 2, but separated by up to $\sim 5 \mathrm{R}_{\mathrm{E}}$. Furthermore, the 2 day period means that the spacecraft orbit can be further phased so that the foot-point of the field line connecting the spacecraft is over European groundbased facilities when the spacecraft are at local noon on every orbit.

Thus the 3-spacecraft fleet sweeps across the dayside magnetopause, remaining in longitudinal alignment, once per orbit. We contend this provides a scientifically highly valuable set of platforms from which to make in situ measurements of the fields and plasma environment. The separation at local noon will vary from $\sim 5 R_{E}$ to near-zero due to orbit precession through the year, providing scientifically highly desirable variations in inter-spacecraft distances. In addition, for more than $50 \%$ of each orbit, the spacecraft will be inside the magnetosphere as they pass through the flanks and the tail. Although not the focus of the science addressed here, this will also provide highly valuable science data for studies of internal magnetospheric processes such as field line resonances, substorm current disruptions and onsets, etc. 


\subsection{Launcher requirements}

IMPALAS could be launched from Kourou using the Soyuz Fregat 2B. A preliminary analysis of the launch scenario suggests that the representative orbits described above can be achieved assuming 3 spacecraft with dry mass of $200 \mathrm{~kg}$ (see Section 5), not including main engine and its subsystems. The 3 IMPALAS spacecraft can be injected into either GTO or GSO by the launcher system, and then proceed under their own propulsion to their individual operational orbits. We have calculated the total launch mass of the IMPALAS mission by considering the delta- $V$ required on each spacecraft to move from the initial orbit provided by the launcher to the operational orbit. This includes first raising all 3 spacecraft to the apogee height of the final elliptical orbit used for 12 and 13 , then making the relevant inclination change and finally circularising the 11 orbit to the required height. Table 3 shows the total delta- $V$ required for these manoeuvres on each spacecraft, and thus the fuel mass required, assuming hydrazine dual propellant. We allow $10 \mathrm{~kg}$ for the main engine mass, and $20 \%$ of the maximum fuel mass requirement to size the fuel tanks. Combining the calculations for each of the 3 spacecraft, we estimate that the mission launch mass is $\sim 2400 \mathrm{~kg}$ for injection into GTO and $1770 \mathrm{~kg}$ for injection into GSO. We note that both these estimates are well within the current lift capability of the Soyuz-Fregat launch system.

\section{Proposed Model Payload}

\subsection{Overview of all proposed payload elements}

The IMPALAS science goals can all be accomplished using a modest payload comprising, with one exception, instruments with a high TRL that have previously been flown with great success on missions such as Cluster and THEMIS. However, alternate concepts providing higher capability and/or reduced resource requirements are currently under development and could be used after qualification. The core payload concept, which should be identical on each of the 3 spacecraft, consists of a DC magnetometer, plasma ion and electron spectrometers and an energetic particle detector. These are supplemented by a nadir-pointing UV auroral imager which would fly only on the 2 spacecraft in inclined orbits. This payload would be controlled by a common payload processor, saving mass and allowing for efficient payload operations. If the resource budgets allow, 2 further instruments, measuring electric fields and ion composition, should be considered as highly desirable.

\subsection{Summary of Core Instruments Key Resources and Characteristics}

\subsubsection{Magnetometer}

Magnetic field vectors are required to fulfil all of the science goals of IMPALAS. They are needed to determine the position of the spacecraft with respect to the magnetopause current layer, identify and characterise flux transfer events, waves, discontinuities, etc. This instrument also supports operation of other payload units, for example by enabling measurement of particle pitch angle distributions. The magnetic field measurements must be made on all 3 spacecraft by a dual sensor fluxgate magnetometer, of the type flown on many previous missions (e.g. Cassini, Cluster, Double Star and THEMIS, thus TRL $=9$ ). Each sensor is comprised of the sensor itself and a near-sensor electronics module. The 2 sensors should both be located on a rigid boom at differing distances from the spacecraft body. These magnetometers will be deployed on all three spacecraft, typically returning field vectors sampled at $10-20 \mathrm{~Hz}$. To return the magnitude and direction of the magnetic field at a rate of $10 \mathrm{~Hz}$, a telemetry rate of $960 \mathrm{bps}$ per sensor will be required, without onboard compression. Typical mass and power requirements for a magnetometer are 1.5 
$\mathrm{kg}$ and $0.5 \mathrm{~W}$ respectively. The orientation of the flux-gate assembly with respect to the spacecraft must be known with a precision of $0.1-0.2$ degrees and a magnetically clean spacecraft will be required to avoid contamination of the magnetic field measurements. Note that, as the spacecraft always remain in the vicinity of the magnetopause, the measurement range is not too variable and the required sensitivity is well within reach of existing instrument technology.

\subsubsection{Dual Sensor Ion and Electron Spectrometer}

Measurements leading to the characterisation of the thermal ion and electron populations in and around the magnetopause current layer are required to fulfil all of the science goals of IMPALAS. They are needed to determine the position of the spacecraft with respect to the magnetopause current layer, identify and characterise FTE's, waves and boundary layers. Measurements of the ion and electron velocity distribution functions (VDF), or at least their moment parameters (density, temperature, flow velocity, etc.) must be made on all three IMPALAS spacecraft with a time resolution of $\sim 3 \mathrm{~s}$. These measurements can be provided by traditional top-hat electrostatic analysers, as commonly flown on space plasma physics missions (e.g. Cassini, Cluster, Double Star), which thus have a TRL of 9 . In such instruments, E/q selection of incoming particles is accomplished through varying an electrostatic potential between two hemispheres, altering the path of incoming particles such that only particles in a narrow energy band can pass through the sensor to impact on MCP detectors. Each sensor typically has a field-of view of a few degrees by 180 degrees. Thus a spin-stabilised spacecraft is required to scan the whole sky and hence measure the full 3D VDF of ions and electrons, from which the basic plasma parameters such as density and bulk velocity can be derived.

These instruments can be deployed on each IMPALAS spacecraft packaged as two dualhead sensor units mounted on opposite sides of each spacecraft. Each unit would have one head configured for measuring electrons and one head configured for measuring ions. Both the ion and electron sensors will be optimised for the more tenuous magnetospheric plasma sampled by the IMPALAS spacecraft, but will include variable geometric factor systems in order to measure denser magnetosheath plasma without saturating the MCP's. Each sensor should have an energy range of a few eV to $\sim 30 \mathrm{keV}$ and an energy resolution of approximately $10-15 \%$. An angular resolution of $10^{\circ} \times 10^{\circ}$ or $20^{\circ} \times 20^{\circ}$ will be sufficient to fulfil the IMPALAS science goals. For an instrument design consisting of two sensor heads for both ions and electrons, a full 3D velocity distribution could be collected every half spin. Thus in order to provide plasma parameters at the required temporal resolution of $\sim 3 \mathrm{~s}$, a spacecraft spin rate of $\sim 10 \mathrm{rpm}$ would be required.

Current conceptual designs for the dual head system (Figure 5) suggest each dual head sensor unit would have a mass of $3 \mathrm{~kg}$ and a power requirement of $3 \mathrm{~W}$. For sensors with an approximate $11^{\circ} \times 11^{\circ}$ angular resolution and 64 energy levels (implying $\sim 15 \%$ energy resolution to cover the full energy range without gaps), a full 3D velocity distribution requires $\sim 256 \mathrm{~kb}$. Thus a data rate of $\sim 86 \mathrm{kbps}$ each is needed for $1 / 2$-spin resolution 3D ion and electron distributions to be telemetered to the ground. Data compression and selection strategies, such as onboard creation of $2 \mathrm{D}$ pitch angle distributions and the onboard calculation of moments, can be implemented to reduce this if necessary. A telemetry rate of $24 \mathrm{kbps}$ is baselined for each species, assuming a conservative compression ratio of 4 and allowing for housekeeping data.

The entrance apertures for each top-hat sensor must be mounted pointing away from the spacecraft body with centre of the fields-of-view perpendicular to the spacecraft spin axis, in order to achieve full $4 \pi$ steradian coverage of the sky every one half spacecraft spin 
period. The each of the dual sensor head units should be mounted on the spacecraft such that the fields-of-view do not include any significant obstructions. The spacecraft should be designed to minimise surface charging effects and emission of spacecraft photo-electrons, particularly near the instrument apertures.

\subsubsection{Energetic Particle Package}

The supra-thermal component of particle distributions is a ubiquitous feature of nonequilibrium, collisionless plasmas including those observed in the near-Earth environment. These populations are most readily described in terms of a kappa function representing a combination of a thermal Maxwellian distribution and a power-law tail. The non-thermal population and rapid field-aligned transport provide the unique capability to remotely sample the acceleration processes and mechanisms taking place within boundaries, and particularly regions of magnetic reconnection. Within the near-Earth plasma environment the non-thermal tail of the distribution is most commonly observed from a few tens of keV and above. The Energetic Particle Package on IMPALAS should thus measure the full 3D ion and electron particle distributions in the energy range from $\sim 20$ to $1000 \mathrm{keV}$, with a temporal cadence of once per spin (i.e. $\sim 6 \mathrm{sec}$ ).

These measurements could be provided on all three spacecraft by an ion implanted silicon based solid state detector of a simple pin hole design, similar to the Imaging Electron Spectrometers employed on Polar, Cluster, THEMIS and Demeter (thus TRL = 9). Incident energetic particles generate electron-hole pairs that produce a signal pulse proportional to the energy of the incident particle. A pulse height distribution is then collected corresponding to the energy spectrum of the energetic particles. Each sensor consists of three detector modules providing an azimuthal slice $\left(20^{\circ} \times 180^{\circ}\right.$ field of view), similar to the plasma spectrometers. Thus a 3D distribution will be built up over the course of a spacecraft spin. The detector will have an energy resolution of $30-40 \%$. Angular resolution depends on the instrument electronics however typical resolutions are of order $10 \times 10$ degrees. The instrument will need to be mounted on the spacecraft such that the detector fan is perpendicular to the spacecraft body, with the centre of the fan perpendicular to the spacecraft spin axis. The FoV should be clear of any spacecraft appendages.

Based on the similar instrument described in the Cross-Scale ASR (Schwartz et al., 2009), we suggest a mass and power envelope of $2 \mathrm{~kg}$ and $2 \mathrm{~W}$ respectively for this instrument. A full 3D distribution consisting of 16 azimuthal angles, 9 polar angles, and 10 energies for both ions and electrons, and returned as 16 bit words at a cadence of once per spin ( $6 \mathrm{~s})$ implies an uncompressed telemetry rate for this instrument of $\sim 7.5 \mathrm{kbps}$. Assuming a conservative compression rate of 4 , and allowing for house-keeping, this instrument requires a telemetry rate of 2 kbps.

\subsubsection{Auroral Imager}

Auroral imagery is necessary to provide context for the in situ measurements from the rest of the payload. Such images essentially provide a remote measurement of activity at the foot points of the magnetic field lines sampled by the spacecraft. The auroral images will be provided by a FUV camera operating in the wavelength range 140-180nm (i.e. the molecular N2 Lyman-Birge-Hopfield emissions), but will not have any spectral capabilities within this wavelength range. UV is necessary in order to capture images of the dayside auroral oval. A concept instrument has been studied for use in lower orbits for the Chinese Kua Fu mission opportunity and a similar concept could be deployed on IMPALAS, installed on the two non-equatorial spacecraft, 12 and 13 only, to provide images of the northern and southern dayside auroral zones concurrently. The instrument would consist of a radially-slumped square-pore MCP focussing optical system, giving a field of view of 
$22.5^{\circ} \times 90^{\circ}$, and a slumped MCP detector. The angular resolution of the instrument is 6 arc minutes (FWHM), providing a spatial resolution of $110 \mathrm{~km}$ for $\mathrm{I} 2$ and $\mathrm{I} 3$ at their perigee. Image acquisition is accomplished using a photon counting system, whereby the detector records the arrival time of each photon. In order to perform aspect reconstruction of photon event data to create an image of the auroral emission, millisecond-resolution time tagging of photon events will be required. The instrument should be mounted on the spacecraft body with the centre of the field of view pointing perpendicular to the spacecraft spin axis (assuming the latter points approximately perpendicular to the ecliptic plane). This ensures that the Earth (and thus the auroral zones) passes through the $\pm 45^{\circ}$ field of view every spacecraft spin at all phases of the orbit. Thus with knowledge of the spacecraft attitude as a function of time an image in an appropriate geophysical coordinate system can be constructed. The image is assembled by integrating photon counts over time, and it is anticipated that an effective integration time of 30 seconds will be compatible with the IMPALAS science requirements. However, time resolution as short as the spacecraft spin period is in principle possible if data storage and telemetry allow. A snapshot from a simulation of the view of the auroral ovals from the 12 and 13 spacecraft is shown in Figure 6 (courtesy S. Milan, University of Leicester).

The camera and associated electronics weigh $5 \mathrm{~kg}$ and consume $15 \mathrm{~W}$ in the configuration defined for Kua Fu. However, for the IMPALAS application, in which the field of view of the instrument can be considerably reduced (potentially to use a single detector MCP, rather than the 4 base-lined for Kua Fu), it is likely that there is scope to reduce these numbers by as much as 50\%. Given the current resolution of the base-lined instrument, the Earth could be viewed in its entirety with an image size of $128 \times 128$ pixels. Using an 8 bit pixel depth implies each image size is $128 \mathrm{kbits}$. At a cadence for image production of 30 seconds, assuming a conservative image compression ratio of 4 and allowing for the telemetry of image metadata and housekeeping, we estimate that the telemetry requirement for this instrument is of order $1.5 \mathrm{kbits} / \mathrm{second}$.

Uniquely among the IMPALAS mission instruments, several elements of the Auroral Imager technology has not previously been flown in space, although concept studies have been carried out at the University of Leicester as part of the work for the Kua Fu mission opportunity. Hence the overall TRL for this instrument is $\sim 4$.

\subsubsection{Common Payload Processor (CPP)}

A common processor should be employed to handle provide all commanding and data buffering and handling functions for all the instruments, including data compression and/or other data reduction activities (for example the generation of 2D pitch angle distributions from the particle instruments if required), thus reducing overall required resources. It will be required to provide instrument functionality control and have the necessary memory and computational resources to receive and decode commands from the spacecraft; provide a buffer for onboard data handling; format, perform lossless compression and transmit instrument science and housekeeping data at a rate depending on spacecraft telemetry mode. The CPP could readily be based on a Field Programmable Gate Array (FPGA) based processor, such as the Leon 3 Fault Tolerant derived processor, and will be able to provide instrument operations, control and perform loss-less data compression. Connectivity to the instruments could be provided by the SpaceWire system. A hot/cold redundant design will be needed since the CPP represents a single point of failure for the whole payload. Based on the Cross-Scale ASR (Schwartz et al., 2009), a mass budget of order $5 \mathrm{~kg}$ and a power budget of $12 \mathrm{~W}$ are required for the CPP. This payload element generates no scientific data, but we baseline $1 \mathrm{kbps}$ of telemetry for housekeeping purposes. 


\subsection{Summary of Highly Desirable Instruments Key Resources and Characteristics}

Although all the science objectives of IMPALAS can be accomplished with the core payload described above, the following instruments will enhance the mission's capabilities and provide added value and further science return.

\subsubsection{D Electric Field Instrument}

By measuring the potential difference between two spherical probes at the end of two wire booms, extending radially from the spacecraft in opposite directions, one can measure a single vector component of the electric field local to the spacecraft. Additional probe pairs mounted orthogonally to the first can be employed to measure additional vector components. The science drivers for the IMPALAS electric field instrument only require a 2D electric field instrument, measuring the electric field components in the spin plane of the spacecraft, which avoids the need for costly and technically challenging spin axis booms. Using the same technique, measuring the potential difference between one or more of the end of boom probes and the spacecraft body provides an estimate of the spacecraft potential, which is a useful proxy for the background plasma density, but also is valuable in accurately reconstructing the true velocity distribution of charged particles (particularly electrons) which may have been modified by the acceleration of particles as they pass through the potential gradient between the spacecraft and the ambient plasma. Both $A C$ and DC electric fields can be measured, DC fields up to a cadence of a few tens of $\mathrm{Hz}$ at a resolution of $0.1 \mathrm{mV} / \mathrm{m}$. Such instruments are capable of sampling the DC electric field at $>100$ samples per second, but this can be reduced or averaged to provide electric field vectors at the lower cadences required for the IMPALAS application.

Similar instruments have flown on THEMIS, Cluster, Polar, Fast and numerous other spacecraft and as such the 2D electric field instrument has a TRL of 9 . Typical instruments consisting of 4 wire boom units have a mass of $\sim 7.5 \mathrm{~kg}$. Total power requirements for the instrument are $\sim 2.5 \mathrm{~W}$. Electric field telemetry requirements for the mission would be comparable to those for the magnetometer.

\subsubsection{Ion Composition Analyser}

Mass discrimination capability, even on one spacecraft in the fleet, would provide significantly better context for the interpretation of all the other measurements. This is because the various physical processes which are the target for the IMPALAS mission on the magnetopause (e.g. magnetic reconnection, boundary wave formation) are known to be mediated by the presence of heavier ions in the system. These may arise, for example, from heavy ion outflow from the dayside auroral zone, and may then populate the regions of space immediately around the magnetopause. Thus, should resources allow, an ion mass spectrometer instrument would be a significant augmentation to the payload. This could be designed to use a common interface to the auroral imager, and then readily deployed in that vacant slot on 11 , which does not need to carry the imager as it is not in an appropriate orbit for viewing the auroral zones. This single spacecraft augmentation would therefore not impact heavily on the desirable common design for each payload bus.

A popular method of mass discrimination is with an electrostatic analyser combined with a time of flight system. After the incoming ions have been energy/charge selected by the electrostatic analyser, they pass through a thin carbon foil which generates a start signal when the resultant electron is detected by a dedicated MCP. The detection of the ion itself by a different MCP provides the stop signal, giving the E/q of the ion. The velocity of the ion through the time of flight system (as determined from the known geometry and the 
difference between the start and stop times) allows the ion $\mathrm{m} / \mathrm{q}$ to be calculated. In this manner $\mathrm{H}^{+}, \mathrm{He}^{+}, \mathrm{He}^{++}$and $\mathrm{O}^{+}$can be distinguished.

The ion composition analyser needed for this application would typically have a mass of $3.5 \mathrm{~kg}$ per sensor and an average power consumption of $3.5 \mathrm{~W}$. A single spin 3D distribution for each of the four ion species measured over 32 energies with angular resolution of $22.5 \mathrm{deg} \times 22.5 \mathrm{deg}$, occupies $128 \mathrm{~kb}$ of memory. Telemetering this data product after compressing by a factor 4 produces a data rate for the instrument of $\sim 6 \mathrm{kbps}$, allowing for housekeeping. Similar instruments have flown on Cluster and STEREO, so the ion composition analyser could be considered to have a TRL of 9 .

\subsection{Payload Summary}

The IMPALAS science payload largely uses proven technology. With the exception of the Auroral Imager, the TRL of the individual instruments is already high, with most critical subsystems already flown in space. A summary of the payload resources is provided in Table 4.

\section{System Requirements and Spacecraft Key Issues}

The 3 IMPALAS spacecraft should be identical in design. As a minimum, they should house the core payload packages described in the previous section. However, I1, the spacecraft bound for the in-ecliptic orbit, need not carry the Auroral Imager due to the poor viewing angle from this orbit. I1 could instead house the highly-desirable ion composition analyser, assuming a common interface slot can be designed for the 2 instruments to avoid non-identical bus design costs. If resources allow the other highly desirable instruments should also be included in the payload.

The IMPALAS spacecraft should be spin-stabilized, similar to the Cluster satellites. We envision the architecture of the spacecraft will resemble a miniaturised Cluster bus, being cylindrical in design. (We note that the core IMPALAS payload is considerably smaller in both number and size of sensors than that of the Cluster mission). Solar panels will be mounted around the curved surfaces of the body to provide power, and the external dimensions of the cylinder will be driven, at least in part, by the need to provide sufficient power to the payload and spacecraft systems. The payload could be mounted on an observation deck at one end of the cylinder in a manner that satisfies the FoV's of the particle sensors. The magnetometer will be mounted on a boom which will be deployed from the observation deck.

The IMPALAS satellite bus should provide command and data handling, telecommunications, attitude control, power systems, thermal control and propulsion. Star-trackers and other attitude control sensors can also be mounted to the observation deck. Other spacecraft systems will be accommodated within the body of the cylinder, as should the fuel tanks and propulsion modules. The heat dissipated in the spacecraft systems and accumulated through the radiation from the Sun can be removed from the spacecraft by a heat pump and radiator system. Critical components of both the payload and spacecraft subsystems may need to be covered by thermal protection material.

We anticipate that all the required system and service components of the spacecraft bus have heritage from previous successful space missions and can be used with only minor modifications. Hence we believe that the TRL of the component comprising the IMPALAS spacecraft bus would range from 7 to 9 . 
The total dry mass of each spacecraft, including the scientific payload, but excluding the propulsion system necessary to achieve orbit insertion, is estimated to be $200.4 \mathrm{~kg}$, including a $30 \%$ margin. The estimated mass distribution is shown in Table 5 . This estimate is based mainly on information contained within the Cross-Scale Assessment Study Report (Schwartz et al., 2009), which considered a number of similar instruments, but more numerous in number than the IMPALAS application. However, we consider that the Cross-Scale bus subsystems will be more than sufficient for the IMPALAS mission and thus provide a conservative estimate of required resources for the latter.

\subsection{Attitude and orbit control}

Monitoring the spin axis orientation and spin phase of the spacecraft requires deployment of a star tracker and sun sensor on each spacecraft. These are required to time attitude control thruster firings and for the operation of instruments. The spin axis must be maintained at the correct attitude for science operations. The pointing knowledge and the accuracy of the spin rate are of $0.1 \mathrm{deg}$ and $1 \%$, respectively. The spacecraft attitude control is achieved using cold gas thrusters. Radial 1-N thrusters can be located at the edge of top and bottom surfaces of the cylindrical spacecraft to optimise their capability. In addition, thrusters directed along the spin-axis are required to provide $\Delta v$ for out-of-plane manoeuvres.

\subsection{On-board data handling and telemetry}

The IMPALAS scientific model payload consists of 5 core sensors with options to include 2 highly desirable instruments. We propose that onboard data handling occurs within the CPP system. In addition to the main data processing and compression functions, the CPP schedules the scientific operations and controls the flow of science data to the spacecraft Data Handling System (DHS). All data exchange and instrument commanding could be done via SpaceWire links from the DHS to the CPP. This approach will afford a decrease in the overall payload mass and readily allow communication between different instruments.

The DHS should contain sufficient mass memory to store 2 entire orbits (4 days) of data. Given the data production rate of the combined core payload (152 kbps) and allowing 2 kbps per spacecraft in housekeeping, this amounts to $\sim 50 \mathrm{Gbits}$.

\subsection{Mission operations concept}

The 3 IMPALAS spacecraft operate in orbits that are phased such that the spacecraft at the same local time at all times. From the ground, they may be separated by up to 60 degrees on the sky. However, twice per orbit (i.e. once per day) the spacecraft will appear, from Earth, to be very close together, when their phased orbits cross the ecliptic plane (the plane of the I1 orbit). Thus a single ground link could be used for all 3 satellites at the same time and hence all 3 satellites could share that communications link. In order to establish feasibility, we have assessed the visibility of the 3 spacecraft from the Kourou ground station $\left(5.3^{\circ} \mathrm{N}, 52.8^{\circ} \mathrm{W}\right)$ for \pm 5 days either side of spring equinox, assuming that the constellation reaches maximum separation at $12 \mathrm{MLT}$ over Scandinavia on that day. The 3 panels of Figure 7 show (black/red trace) the angle from the zenith and (blue/light blue trace) the distance to each of the 3 spacecraft from the ground station. Periods in which the spacecraft are above 15 degrees from the horizon are designated with the red and light blue line sections. This shows the communications link to Kourou for the IMPALAS satellites could be available for $\sim 40 \%$ of the orbit, or in continuous periods of $\sim 19$ hours per 2 day orbit. During these periods, the 3 spacecraft range in distance from $9.0-10.5$ $R_{E}$ from the ground station. 
The downlink requirement for the mission (3 satellites) is $\sim 12.5$ Gbits of data per day. Communications could be direct to ground stations using an X-band system such as that base-lined for GAIA. This provides variable data-rates up to $\sim 6.5 \mathrm{Mbps}$. Hence transmission of scientific data from the mission takes a combined $\sim 1.2 \mathrm{hrs}$ per day.

These simple calculations demonstrate that it is entirely feasible to return the full IMPALAS dataset to a single ground station at this rate, using less than $10 \%$ of the available communications window for each spacecraft.

\subsection{Estimated overall resources}

The overall mass (see Table 3 ) is estimated as $2400 \mathrm{~kg}$ (for launch into an initial GTO orbit) or $1770 \mathrm{~kg}$ (for launch into an initial GSO orbit) and includes the 3 satellites, as well as the fuel for injection into the required orbit and changing the inclinations. This also includes $30 \%$ margins for the bus and payload (see Table 5). The dimensions of the IMPALAS spacecraft will need to be chosen to comply with the fairing of the Soyuz Fregat 2B when the 3 spacecraft are stacked on top of each other inside the fairing. The power requirement for each spacecraft is of order $350 \mathrm{~W}$, including $30 \%$ margin, for the bus and payload subsystems (Table 5). The entire IMPALAS constellation downlinks daily about 1.7 Gbits of scientific data (Table $4+20 \%$ margin), which is realistic using the X-band antennas at ESA ground stations.

\subsection{Specific environmental constraints}

The spacecraft must be magnetically clean. This means that intrinsically magnetic as well as magnetically soft materials should not be used in its construction or within the payload components. A program to document the magnetic activity on the spacecraft should be carried out prior to launch and steps taken to reduce residual magnetic fields of spacecraft origin at the position of the magnetometer on the boom. The outer surface of the spacecraft must also be electrically conducting to avoid differential surface charging and to provide electrically clean environment for low-energy electron and ion measurements (and electric field measurements if flown). Instruments containing MCP's (ion and electron spectrometers, auroral imager) require a vacuum in which to operate. These instruments must be maintained under constant dry nitrogen purge until launch. Before power-up of the instruments on-orbit, a period of time will be required to allow evacuation of gas from the instrument volume (via specific out-gassing apertures in the structure in the case of the auroral imager). No instrument should be placed in the path of gas venting from another.

\section{Technology Development, Programmatics and Cost}

\subsection{Technology Development requirements}

Since most of the required payload listed above currently exists in a form that would meet the measurement requirements likely to be imposed by the flow-down from the science goals, there are no significant technical issues likely to arise from the payload. However, the mission could be seen as providing a flight opportunity for next generation instruments, such that some technology development should be accommodated in this area. The Auroral Imager proposed for the mission is a notable exception to the overall level of payload readiness. Although MCPs and filters have $T R L=9$, some elements of the MCP readout and optics are currently TRL 4-5. However, elevating the TRL of the instrument to an acceptable level depends on developing only one or two of its subsystems.

No novel technologies are necessary for the development of the spacecraft bus or the mission operation. The spacecraft and their required subsystems have high heritage 
within industry. The spacecraft will need to undergo a magnetic and electrostatic cleanliness program. In addition, a means of stacking the set of 3 spacecraft within the launcher fairing, together with a mechanism for their dispensing after reaching GTO/GSO orbit, will need to be developed by industry. A full optimisation of the orbit parameters will be required, together with analysis of the options for launch and delivery of the spacecraft to their 3 distinct operational orbits from a single launch. Otherwise this mission concept will require a standard development for a small satellite measuring fields and plasmas in near-Earth space.

\subsection{Overall mission cost analysis}

In order to demonstrate the feasibility of the IMPALAS mission within the ESA M-class cost envelope, we make direct comparison to the Cross-Scale assessment study (Schwartz et al., 2009). The Cross-Scale mission report considered a fleet of 7 spacecraft, carrying a total of 107 instruments. In contrast, the IMPALAS mission proposed here consists of 3 spacecraft carrying a total of 17 sensor units. Comparison to Cluster, which flew 72 sensor units on 4 spacecraft, could also be made. Although we recognize that there are one-off costs (launch, initial spacecraft design, ESA study costs) applicable to both missions, we contend that the relative sizes of the IMPALAS mission described here to the ESA-assessed Cross-Scale mission imply the former will require less than half the recurrent industrial build costs and a small fraction of the operating costs. Since ESA estimated the cost of Cross-Scale to be $\sim 600 \mathrm{ME}$ in December 2009, we conclude that the IMPALAS mission will easily fit within the $475 \mathrm{ME}$ cost cap set for the M3 opportunity.

\subsection{Mission Schedule Drivers, Risks and Alternate Strategies}

IMPALAS is a modest and relatively low-risk multi-spacecraft mission based largely on flight-proven spacecraft technology and instrumentation. There are no significant developments required that could significantly impact the schedule once the mission has kicked-off and the instrument design and build phases funded. Nevertheless, for both financial reasons and in consideration of in flight spacecraft failure, it is important to assess the extent to which the science objectives could be addressed with fewer spacecraft. Firstly we note that a single spacecraft mission most likely cannot add significantly to what has been achieved by previous missions. Indeed, given the success of the Cluster mission (4 spacecraft flying in relatively close formation compared to the proposed IMPALAS separations), it is unlikely that a single spacecraft mission will add anything at all to current understanding. Failure of a single spacecraft, or a descope to provide a dual spacecraft mission, will provide very useful conjunctions for which a subset of the science goals would be achievable. Primary loss (depending on which spacecraft is lost) will be the ability to make simultaneous dual hemispheric measurements at the magnetopause with the consequent failure to meet the related science goals. Finally, if resources, providing a $4^{\text {th }}$ spacecraft, identical to $I 1$ and in the same equatorial orbit, but a few hours ahead or behind that spacecraft, will allow the scientific studies described herein to be extended to the second dimension along the magnetopause surface, and thus increase the overall scientific return.

\section{Summary}

In this paper we have described a medium-sized mission to carry a scientific payload to measure fields and plasmas (including magnetometer, ion and electron spectrometer and energetic particle telescopes) on 3 identical spacecraft in synchronized orbits. These orbits provide inter-spacecraft separations of $\sim 5 R_{E}$ along the direction of dayside terrestrial field lines as the 3 spacecraft skim the dayside magnetopause and continuously 
sampling this boundary over periods of many hours. A key feature of these orbits are that they are phased such that (i) all 3 spacecraft maintain common longitude and thus sample along the same magnetopause field line; (ii) the 3 spacecraft reach local midday when northern European or other ground-based facilities also lie near local midday, enabling simultaneous sampling of magnetopause field lines and their footprints.

This mission concept would provide an unprecedented level of access to the terrestrial dayside magnetopause, which is the primary site of energy transfer from the solar wind into the magnetosphere. Specific plasma processes operating on the magnetopause include magnetic reconnection, generation of boundary waves, propagation of pressurepulse induced deformations of the boundary, formation of boundary layers and generation of Alfven waves and field-aligned current systems connecting the boundary to the inner magnetosphere and ionosphere. However, many of the details of these processes are not fully understood, and their relevance to the global dynamics of the magnetospheric system remains unresolved. Measurements which would be provided by the mission concept described here would be decisive in resolving these issues.

\section{References}

Amm, O., Grocott, A., Lester, M., Yeoman, T.K.: Local determination of ionospheric plasma convection from coherent scatter radar data using the SECS technique. J Geophys Res-Space 115, A03304 (2010). doi: 10.1029/2009ja014832

Angelopoulos, V., The THEMIS Mission, Space Sci. Rev., 141, 5-34, (2008), doi:10.1007/s11214-008-9336-1

Chisham, G., Pinnock, M., A. S. Rodger, A.S. and Villain, J.-P., High-time resolution conjugate SuperDARN radar observations of the dayside convection response to changes in IMF, Ann. Geophys., 18, 191-201, (2000)

Chisham, G., Freeman, M. P., Coleman, I. J., Pinnock, M., Hairston, M. R., Lester, M., and Sofko, G.: Measuring the dayside reconnection rate during an interval of due northward interplanetary magnetic field, Ann. Geophys., 22, 4243-4258, (2004). doi:10.5194/angeo-22-4243-2004

Cooling, B.M.A., Owen, C.J., Schwartz, S.J.: Role of the magnetosheath flow in determining the motion of open flux tubes. J Geophys Res-Space 106(A9), 18763$18775(2001)$

Cowley, S.W.H., Owen, C.J.: A Simple Illustrative Model of Open Flux Tube Motion over the Dayside Magnetopause. Planet Space Sci 37(11), 1461-1475 (1989)

Denton, M.H., Borovsky, J.E.: Superposed epoch analysis of high-speed-stream effects at geosynchronous orbit: Hot plasma, cold plasma, and the solar wind. J Geophys Res-Space 113(A7), A07216 (2008). doi:10.1029/2007ja012998

Dungey, J.W.: Interplanetary Magnetic Field and Auroral Zones. Phys Rev Lett 6(2), 47 (1961)

Elphic, R. C., Observations of Flux Transfer Events: A Review, in Physics of the Magnetopause, Geophysical Monograph Series, Volume 90, ISBN 0-87590-047-X, p225 (1995).

Fairfield, D.H., Average and Unusual Locations of Earths Magnetopause and Bow Shock. J Geophys Res 76(28), 6700 (1971)

Fear, R.C., Milan, S.E., Fazakerley, A.N., Fornacon, K.H., Carr, C.M., Dandouras, I.: Simultaneous observations of flux transfer events by THEMIS, Cluster, Double Star, and SuperDARN: Acceleration of FTEs. J Geophys Res-Space 114, A10213 (2009). doi:10.1029/2009ja014310

Fuselier, S.A., Trattner, K.J., Petrinec, S.M., Owen, C.J., Reme, H.: Computing the reconnection rate at the Earth's magnetopause using two spacecraft observations. J Geophys Res-Space 110(A6), A06212 (2005). doi:10.1029/2004ja010805 
Gosling, J.T., Thomsen, M.F., Bame, S.J., Onsager, T.G., Russell, C.T.: The Electron Edge of the Low Latitude Boundary-Layer during Accelerated Flow Events. Geophys Res Lett 17(11), 1833-1836 (1990)

Hasegawa, H., Retino, A., Vaivads, A., Khotyaintsev, Y., Andre, M., Nakamura, T.K.M., Teh, W.L., Sonnerup, B.U.O., Schwartz, S.J., Seki, Y., Fujimoto, M., Saito, Y., Reme, H., Canu, P.: Kelvin-Helmholtz waves at the Earth's magnetopause: Multiscale development and associated reconnection. J Geophys Res-Space 114, A12207 (2009). doi:10.1029/2009ja014042

Horbury, T.S., Lucek, E.A.: Size, shape, and orientation of magnetosheath mirror mode structures. J Geophys Res-Space 114, A05217 (2009). doi:10.1029/2009ja014068

Kuznetsova, M.M., Sibeck, D.G., Hesse, M., Wang, Y., Rastaetter, L., Toth, G., Ridley, A.: Cavities of weak magnetic field strength in the wake of FTEs: Results from global magnetospheric MHD simulations. Geophys Res Lett 36, L10104 (2009). doi:10.1029/2009gl037489

Milan, S.E., Cowley, S.W.H., Lester, M., Wright, D.M., Slavin, J.A., Fillingim, M., Carlson, C.W., Singer, H.J.: Response of the magnetotail to changes in the open flux content of the magnetosphere. J Geophys Res-Space 109(A4), A04220 (2004). doi:10.1029/2003ja010350

Milan, S.E., Lester, M., Cowley, S.W.H., Oksavik, K., Brittnacher, M., Greenwald, R.A., Sofko, G., Villain, J.P.: Variations in the polar cap area during two substorm cycles. Ann Geophys-Germany 21(5), 1121-1140 (2003)

Nakamura, R., Retino, A., Baumjohann, W., Volwerk, M., Erkaev, N., Klecker, B., Lucek, E.A., Dandouras, I., Andre, M., Khotyaintsev, Y.: Evolution of dipolarization in the near-Earth current sheet induced by Earthward rapid flux transport. Ann GeophysGermany 27(4), 1743-1754 (2009)

Nakamura, T.K.M., Fujimoto, M., Otto, A.: Structure of an MHD-scale Kelvin-Helmholtz vortex: Two-dimensional two-fluid simulations including finite electron inertial effects. J Geophys Res-Space 113(A9), A09204 (2008). doi:10.1029/2007ja012803

Nakamura, T.K.M., Hasegawa, H., Shinohara, I.: Kinetic effects on the Kelvin-Helmholtz instability in ion-to-magnetohydrodynamic scale transverse velocity shear layers: Particle simulations. Phys Plasmas 17(4), 042119 (2010). doi:10.1063/1.3385445 Owen, C.J., Marchaudon, A., Dunlop, M.W., Fazakerley, A.N., Bosqued, J.M., Dewhurst, J.P., Fear, R.C., Fuselier, S.A., Balogh, A., Reme, H.: Cluster observations of "crater" flux transfer events at the dayside high-latitude magnetopause. J Geophys Res-Space 113(A7), A07s04 (2008). doi:10.1029/2007ja012701

Paschmann, G., Haerendel, G., Papamastorakis, I., Sckopke, N., Bame, S.J., Gosling, J.T., Russell, C.T.: Plasma and Magnetic-Field Characteristics of Magnetic-Flux Transfer Events. J Geophys Res-Space 87(Na4), 2159-2168 (1982)

Paularena, K.I., Richardson, J.D., Kolpak, M.A., Jackson, C.R., Siscoe, G.L.: A dawn-dusk density asymmetry in Earth's magnetosheath. J Geophys Res-Space 106(A11), 25377-25394 (2001)

Phan, T.D., Paschmann, G., Baumjohann, W., Sckopke, N., Luhr, H.: The Magnetosheath Region Adjacent to the Dayside Magnetopause - AMPTE/IRM Observations. J Geophys Res-Space 99(A1), 121-141 (1994)

Raeder, J.: Flux Transfer Events: 1. generation mechanism for strong southward IMF. Ann Geophys-Germany 24(1), 381-392 (2006)

Rijnbeek, R.P., Cowley, S.W.H., Southwood, D.J., Russell, C.T.: A Survey of Dayside Flux-Transfer Events Observed by Isee-1 and Isee-2 Magnetometers. J Geophys Res-Space 89(Na2), 786-800 (1984)

Russell, C.T., Elphic, R.C.: Initial Isee Magnetometer Results - Magnetopause Observations. Space Sci Rev 22(6), 681-715 (1978) 
Russell, C.T., Elphic, R.C.: Isee Observations of Flux-Transfer Events at the Dayside Magnetopause. Geophys Res Lett 6(1), 33-36 (1979)

Schwartz, S. J., Horbury, T.S., Owen, C.J., Baumjohann, W., Nakamura, R., Canu, P., Roux, A., Sahraoui, F., Louarn, P., Sauvaud, J.-A., Pincon, J.-L., Vaivads, A., Marcucci, M.F., Anastasiadis, A., Fujimoto, M., Escoubet, C.P., Taylor. M.G.G.T., Eckersley, S., Allouis, E., Perkinson, M.-C., Cross-Scale: Multi-scale Coupling in Space Plasmas, Cosmic Visions 2007 special issue of Exp. Astron., (Astrophysical Instrumentation and Methods), doi: 10.1007/s10686-008-9085-x, 2008.

Schwartz, S.J., Bale, S.D., Fujimoto, M., Hellinger, P., Le, G., Liu, W., Louarn, P., Mann, I., Nakamura, R., Owen, C.J., Pinçon, J.-L.,Sorriso-Valvo, L., Vaivads, A., WimmerSchweingruber, R.F., Falkner, P., Wielders, A., Escoubet, C. P., Taylor, M., and Masson, A., Multi-Scale Coupling in Space Plasmas, Cross-Scale Assessment Study Report (ESA/SRE-2009-1), ESA, 01 Dec 2009.

Sibeck, D.G.: A Model for the Transient Magnetospheric Response to Sudden Solar-Wind Dynamic Pressure Variations. J Geophys Res-Space 95(A4), 3755-3771 (1990)

Sibeck, D.G., Borodkova, N.L., Schwartz, S.J., Owen, C.J., Kessel, R., Kokubun, S., Lepping, R.P., Lin, R., Luhr, H., McEntire, R.W., Meng, C.I., Mukai, T., Nemecek, Z., Parks, G., Phan, T.D., Romanov, S.A., Safrankova, J., Sauvaud, J.A., Singer, H.J., Solovyev, S.I., Szabo, A., Takahashi, K., Williams, D.J., Yumoto, K., Zastenker, G.N.: Comprehensive study of the magnetospheric response to a hot flow anomaly. J Geophys Res-Space 104(A3), 4577-4593 (1999)

Sibeck, D.G., Kudela, K., Mukai, T., Nemecek, Z., Safrankova, J.: Radial dependence of foreshock cavities: a case study. Ann Geophys-Germany 22(12), 4143-4151 (2004)

Smith, M.F., Owen, C.J.: Temperature Anisotropies in a Magnetospheric Fte. Geophys Res Lett 19(19), 1907-1910 (1992)

Smith, M.F., Rodgers, D.J.: Ion Distributions at the Dayside Magnetopause. J Geophys Res-Space 96(A7), 11617-11624 (1991)

Sonnerup, B.U.O., Hasegawa, H., Paschmann, G.: Anatomy of a flux transfer event seen by Cluster. Geophys Res Lett 31(11), L11803 (2004). doi:10.1029/2004gl020134

Spanswick, E., Reeves, G.D., Donovan, E., Friedel, R.H.W.: Injection region propagation outside of geosynchronous orbit. J Geophys Res-Space 115, A11214 (2010). doi:10.1029/2009ja015066

Thomsen, M.F., Stansberry, J.A., Bame, S.J., Fuselier, S.A., Gosling, J.T.: Ion and Electron Velocity Distributions within Flux-Transfer Events. J Geophys Res-Space 92(A11), 12127-12136 (1987)

Trattner, K.J., Mulcock, J.S., Petrinec, S.M., Fuselier, S.A.: Probing the boundary between antiparallel and component reconnection during southward interplanetary magnetic field conditions. J Geophys Res-Space 112(A8), A08210 (2007). doi:10.1029/2007ja012270 


\begin{tabular}{|l|l|}
\hline \multicolumn{1}{|c|}{ Question: } & \multicolumn{1}{|c|}{ How solved: } \\
\hline $\begin{array}{l}\text { What is the location of the MP } \\
\text { reconnection site for given conditions? }\end{array}$ & $\begin{array}{l}\text { Large number of measurements of particle } \\
\text { dispersions/cut-offs at different locations along the } \\
\text { same reconnected field line. }\end{array}$ \\
\hline $\begin{array}{l}\text { What is the importance of FTE's in global } \\
\text { dynamics of the magnetosphere? }\end{array}$ & $\begin{array}{l}\text { Determine if FTE's appear in only one or in both } \\
\text { hemispheres simultaneously (adding open flux or not?). }\end{array}$ \\
\hline $\begin{array}{l}\text { How do boundary waves evolve as they } \\
\text { propagate across the magnetopause? }\end{array}$ & $\begin{array}{l}\text { Regular multi-point observations of boundary } \\
\text { deformations at different distances from their origin. }\end{array}$ \\
\hline $\begin{array}{l}\text { Which mechanisms form boundary layers } \\
\text { at the MP and how do they vary or evolve } \\
\text { with position? }\end{array}$ & $\begin{array}{l}\text { Regular and simultaneous multi-point observations of } \\
\text { boundary layers across the dayside MP. }\end{array}$ \\
\hline $\begin{array}{l}\text { How do disturbances, discontinuities and } \\
\text { waves propagate within the } \\
\text { magnetosheath and how and where can } \\
\text { they impact the MP? }\end{array}$ & $\begin{array}{l}\text { Widely spaced measurements within the } \\
\text { magnetosheath at times when the magnetosphere is } \\
\text { compressed and MP is located below average position. }\end{array}$ \\
\hline $\begin{array}{l}\text { How do MP disturbances of all types } \\
\text { propagate along field lines and into the } \\
\text { ionosphere? }\end{array}$ & $\begin{array}{l}\text { Multi-point measurements of Alfvènic disturbances and } \\
\text { field aligned currents along the same field line, } \\
\text { combined with regular observations of those field line } \\
\text { foot-points by ground-based facilities. }\end{array}$ \\
\hline
\end{tabular}

Table 1: Examples of top level science objectives addressed by the IMPALAS mission concept.

\begin{tabular}{|c|c|c|}
\hline Required Measurement & $\begin{array}{c}\text { Required } \\
\text { Instruments }\end{array}$ & Required for: \\
\hline $\begin{array}{l}\text { 3D magnetic field vector @ 1 sec } \\
\text { resolution }\end{array}$ & Magnetometer & $\begin{array}{l}\text { Identification of MP crossings, plasma } \\
\text { waves and FTE's, Walen tests for } \\
\text { identification of reconnection outflows }\end{array}$ \\
\hline $\begin{array}{l}\text { 3D velocity distribution functions of } \\
\text { electrons and ions, few eV to } \sim 30 \mathrm{keV} @ \\
\sim 3 \mathrm{sec} \text { (half spin) resolution. }\end{array}$ & $\begin{array}{l}\text { Ion \& Electron } \\
\text { Spectrometers }\end{array}$ & $\begin{array}{l}\text { Calculations of plasma moments } \\
\text { (density, velocity, temperature, } \\
\text { pressure), identification of MP } \\
\text { crossings, particle cut-offs to locate } \\
\text { reconnection site, Walen tests for } \\
\text { identification of reconnection outflows. }\end{array}$ \\
\hline $\begin{array}{l}\text { 3D velocity distribution function of energetic } \\
\text { ion and electrons, }>30 \mathrm{keV} @ \sim 6 \mathrm{sec} \\
\text { (spin) resolution. }\end{array}$ & $\begin{array}{l}\text { Energetic } \\
\text { Particle } \\
\text { Detectors } \\
\end{array}$ & $\begin{array}{l}\text { Determine boundary motions and } \\
\text { identify particle acceleration } \\
\text { signatures. }\end{array}$ \\
\hline $\begin{array}{l}\text { Imaging of auroral dynamics at foot point of } \\
\text { magnetic field line threading spacecraft } \\
\text { locations @ } 30 \text { sec cadence. High } \\
\text { inclination orbits only. }\end{array}$ & $\begin{array}{l}\text { Auroral Zone } \\
\text { Imager }\end{array}$ & $\begin{array}{l}\text { Provides context and additional link } \\
\text { between in situ and ground-based } \\
\text { measurements. }\end{array}$ \\
\hline Desired Measurements & $\begin{array}{c}\text { Desirable } \\
\text { Instruments }\end{array}$ & Desired for: \\
\hline $\begin{array}{l}2 \mathrm{D} \text { electric field vector } @ \sim 1 \mathrm{sec} \text { resolution } \\
\left(3^{\text {rd }} \text { component derived from } \mathrm{E} . \mathrm{B}=0\right) \text {. }\end{array}$ & $\begin{array}{l}\text { Electric field } \\
\text { booms }\end{array}$ & $\begin{array}{l}\text { Identification of plasma waves, } \\
\text { measurement of convection electric } \\
\text { fields within boundary layers, } \\
\text { measurement of s/c potential, total } \\
\text { plasma density }\end{array}$ \\
\hline $\begin{array}{l}\text { Measurement of composition of ambient } \\
\text { thermal plasma @ } \sim 6 \sec \text { (spin) resolutio }\end{array}$ & $\begin{array}{l}\text { Ion Mass } \\
\text { Spectrometer }\end{array}$ & $\begin{array}{l}\text { Plasma composition for correct } \\
\text { analyses, tracers of particle origins } \\
\text { within boundary layers; }\end{array}$ \\
\hline
\end{tabular}

Table 2: Strawman payload for the IMPALAS mission concept. 


\begin{tabular}{|l|c|c|}
\hline Spacecraft Details & GTO & GSO \\
\hline Engine Specific Impulse & 270 & 270 \\
\hline Dry Mass (kg) - See Section 5. & 200 & 200 \\
\hline Fuel for operations (kg) & 20 & 20 \\
\hline Mass to orbit (kg) & 220 & 220 \\
\hline Initial orbit (provided by launcher) & & \\
\hline Apogee altitude (km) & 35768 & 35768 \\
\hline Perigee Altitude (km) & 250 & 35768 \\
\hline Inclination (deg) & 7 & 0 \\
\hline
\end{tabular}

Total velocity change for raising 3 s/c to elliptic operational orbit, individually changing inclination, then circularising the $\mathbf{I}$ orbit

\begin{tabular}{|l|c|c|}
\hline Total Delta V, I2 (km/s) & 3.46 & 2.70 \\
\hline Total Delta V, I3 (km/s) & 2.17 & 0.90 \\
\hline Total Delta V, I1 (km/s) & 3.06 & 2.57 \\
\hline Fuel and propulsion system masses required & & \\
\hline Fuel Required, I2 (kg) & 591.9 & 388.8 \\
\hline Fuel Required, I3 (kg) & 279.1 & 89.6 \\
\hline Fuel Required, I1 (kg) & 478.2 & 361.1 \\
\hline Engine Mass (per spacecraft) & 10.0 & 10.0 \\
\hline Fuel Tank Mass (per spacecraft, 20\% max fuel mass, kg) & 118.4 & 77.8 \\
\hline Total Launch Mass (3 s/c + fuel, $\mathbf{~ k g ) ~}$ & $\mathbf{2 3 9 4 . 4}$ & $\mathbf{1 7 6 2 . 7}$ \\
\hline
\end{tabular}

Table 3: Launch Mass Estimates for the IMPALAS mission, assuming initial injection by the launcher system into GTO or GSO.

\begin{tabular}{|c|c|c|c|c|c|c|}
\hline \multicolumn{2}{|c|}{$\begin{array}{c}\text { Required } \\
\text { Instruments }\end{array}$} & Mass & Power & $\begin{array}{l}\text { Telemetry } \\
\text { rate }\end{array}$ & Volume & $\begin{array}{l}\text { Source / } \\
\text { Reference }\end{array}$ \\
\hline \multicolumn{2}{|c|}{ Magnetometer } & $1.5 \mathrm{~kg}$ & $0.5 \mathrm{~W}$ & $1 \mathrm{kbps}$ & $11 \times 5 \times 5 \mathrm{~cm}^{3}$ & Cluster, Cassini \\
\hline \multicolumn{2}{|c|}{$\begin{array}{c}\text { Ion \& Electron } \\
\text { Spectrometers }(x 2)\end{array}$} & $6 \mathrm{~kg}$ & $6 \mathrm{~W}$ & $\begin{array}{c}24 \text { kbps (ions) } \\
24 \text { kbps } \\
\text { (electrons) }\end{array}$ & $26 \times 15 \times 26 \mathrm{~cm}^{3}$ & $\begin{array}{l}\text { NASA MMS / Cross } \\
\text { Scale ASR/ Solar } \\
\text { Orbiter SWA/EAS }\end{array}$ \\
\hline \multicolumn{2}{|c|}{$\begin{array}{l}\text { Energetic Particle } \\
\text { Detector }\end{array}$} & $2 \mathrm{~kg}$ & $2 \mathrm{~W}$ & $\begin{array}{l}2 \text { kbps (ions } \\
\text { and electrons) }\end{array}$ & $20 \times 10 \times 20 \mathrm{~cm}^{3}$ & Cross Scale ASR \\
\hline \multicolumn{2}{|c|}{$\begin{array}{l}\text { Auroral Zone } \\
\text { Imager }^{1}\end{array}$} & $5 \mathrm{~kg}$ & $15 \mathrm{~W}$ & $1.5 \mathrm{kbps}$ & $10 \times 20 \times 15 \mathrm{~cm}^{3}$ & Kua Fu Study \\
\hline \multicolumn{2}{|c|}{$\begin{array}{c}\text { Common Payload } \\
\text { Processor }\end{array}$} & $5 \mathrm{~kg}$ & $12 \mathrm{~W}$ & $1 \mathrm{kbps}(\mathrm{HK})$ & $20 \times 12 \times 10 \mathrm{~cm}^{3}$ & $\begin{array}{l}\text { Themis CPP } \\
\text { (supporting } 5 \\
\text { instruments) }\end{array}$ \\
\hline \multirow{2}{*}{ TOTAL } & 12,13 & $19.5 \mathrm{~kg}$ & $35.3 \mathrm{~W}$ & $54.5 \mathrm{kbps}$ & & \\
\hline & 11 & $14.5 \mathrm{~kg}$ & $20.3 \mathrm{~W}$ & 53 kbps & & \\
\hline \multicolumn{7}{|c|}{$\begin{array}{c}\text { Desirable } \\
\text { Instruments }\end{array}$} \\
\hline \multicolumn{2}{|c|}{$\begin{array}{l}\text { Electric field } \\
\text { booms }\end{array}$} & $7.5 \mathrm{~kg}$ & $2.5 \mathrm{~W}$ & $1 \mathrm{kbps}$ & $20 \times 15 \times 30 \mathrm{~cm}^{3}$ & Cross Scale ASR \\
\hline \multicolumn{2}{|c|}{$\begin{array}{c}\text { Ion Mass } \\
\text { Spectrometer }^{2} \\
\end{array}$} & $3.5 \mathrm{~kg}$ & $6 \mathrm{~W}$ & $6 \mathrm{kbps}$ & $20 \times 30 \times 20 \mathrm{~cm}^{3}$ & Cross Scale ASR \\
\hline \multicolumn{7}{|c|}{$\begin{array}{l}\text { Table 4: IMPALAS Strawman payload resource envelopes. } \\
{ }_{1}^{1} \text { Auroral Imager to be flown on I } 2 \text { and I3 only; }\end{array}$} \\
\hline
\end{tabular}




\begin{tabular}{|c|c|c|c|c|}
\hline System & Subsystem & $\begin{array}{c}\text { Mass } \\
(\mathrm{kg})\end{array}$ & $\begin{array}{l}\text { Power } \\
\text { (W) }\end{array}$ & Source \\
\hline \multirow{10}{*}{$\begin{array}{c}\text { Spacecraft } \\
\text { Bus }\end{array}$} & Structure & 41.8 & 116.8 & \multirow{10}{*}{$\begin{array}{c}\text { Cross } \\
\text { Scale } \\
\text { ASR, } \\
\text { Solution } \\
2\end{array}$} \\
\hline & Thermal Control & 4.6 & 0.0 & \\
\hline & Mechanism & 6.6 & \multirow{3}{*}{22.2} & \\
\hline & Communication & 11.7 & & \\
\hline & Data Handling & 6.6 & & \\
\hline & AOCS & 3.6 & 4.3 & \\
\hline & Propulsion & 11.5 & 5.5 & \\
\hline & Power & 38.3 & 16.8 & \\
\hline & Harness & 6.0 & 0.0 & \\
\hline & RF system & 4.0 & 68 & \\
\hline $\begin{array}{l}\text { Science } \\
\text { Payload }\end{array}$ & Instruments & 19.5 & 35.3 & $\begin{array}{c}\text { Section } \\
4, \text { Table } \\
4.1\end{array}$ \\
\hline Subtotal & & 154.20 & 268.90 & \\
\hline ESA Margin & $30 \%$ & 46.2 & 80.7 & \\
\hline TOTAL & & $\begin{array}{c}200.4 \\
\mathrm{~kg}\end{array}$ & $349.6 \mathrm{~W}$ & \\
\hline
\end{tabular}

Table 5: Mass and Power requirements for the IMPALAS spacecraft bus, without fuel and engine subsystems 

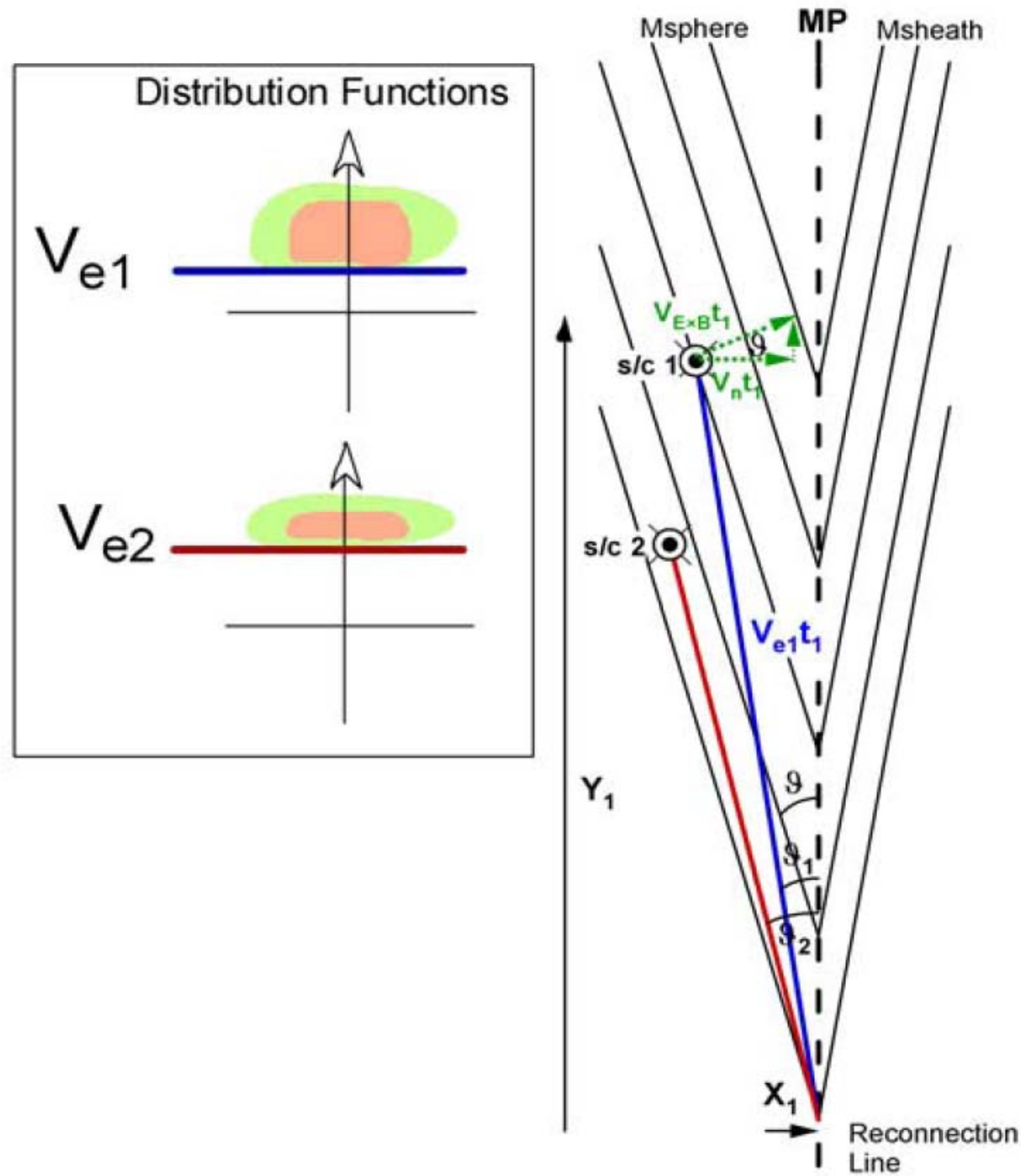

Figure 1: Two-dimensional geometry used to compute the inflow velocity and the distance to the reconnection line given two spacecraft observations in the reconnection layer. For the spacecraft locations in the layer, the velocity distributions in the spacecraft reference frame will resemble those in the inset. In particular, the cut-off velocity $\left(\mathrm{V}_{\mathrm{e} 1}\right)$ for spacecraft 1 will be lower than that for spacecraft $2\left(V_{\mathrm{e} 2}\right)$ because spacecraft 2 is closer to the edge of the reconnection layer (defined as the magnetic field line directly connected to the reconnection line). The blue and red lines emanating from the reconnection site show the trajectories of these ions moving at the cut-off velocity (from Fuselier et al., 2005, Copyright AGU)). 

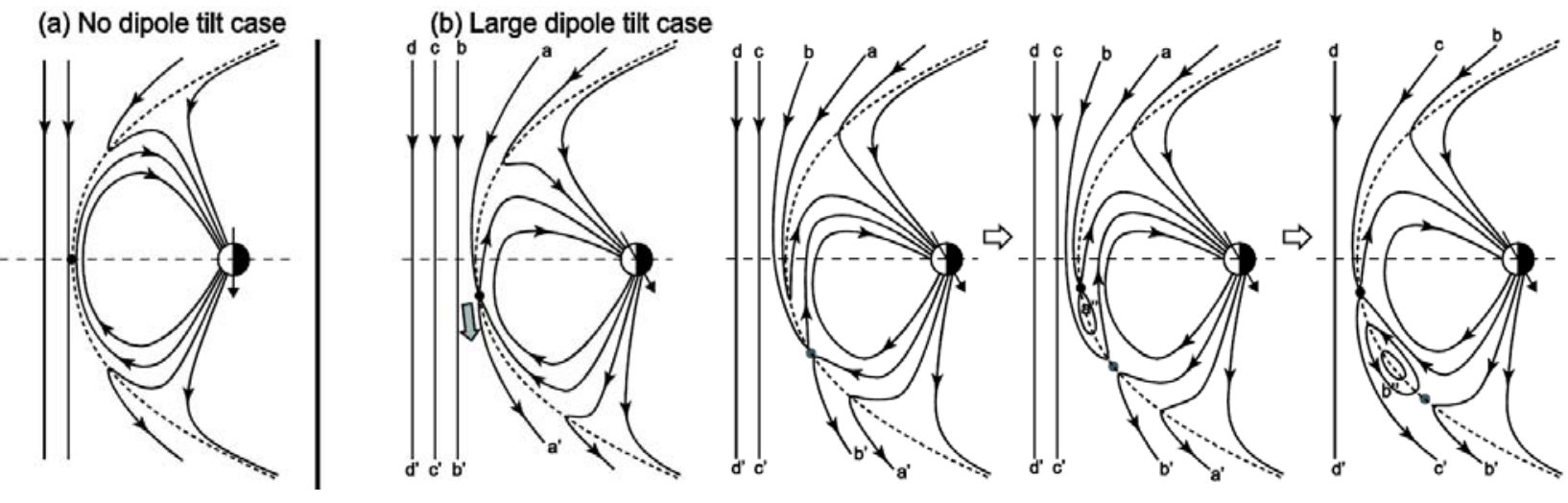

Figure 2: The IMPALAS mission will confirm, or otherwise, the importance of FTE's in global dynamics of the magnetosphere. The observations will help determine whether FTE's play a significant role in the addition of flux to the magnetotail (formed by reconnection between IMF and closed terrestrial field lines, similar to case (a)), or whether they are simply a restructuring of already reconnected field lines caused by dipole tilt effects (as in case (b)), as suggested by e.g. Raeder (2006). IMPALAS will resolve this issue by discovering if matched FTE's appear in both hemispheres simultaneously or as single structures appearing in one hemisphere only (and thus if they add net open flux to the magnetosphere or not). (Copyright AGU) 


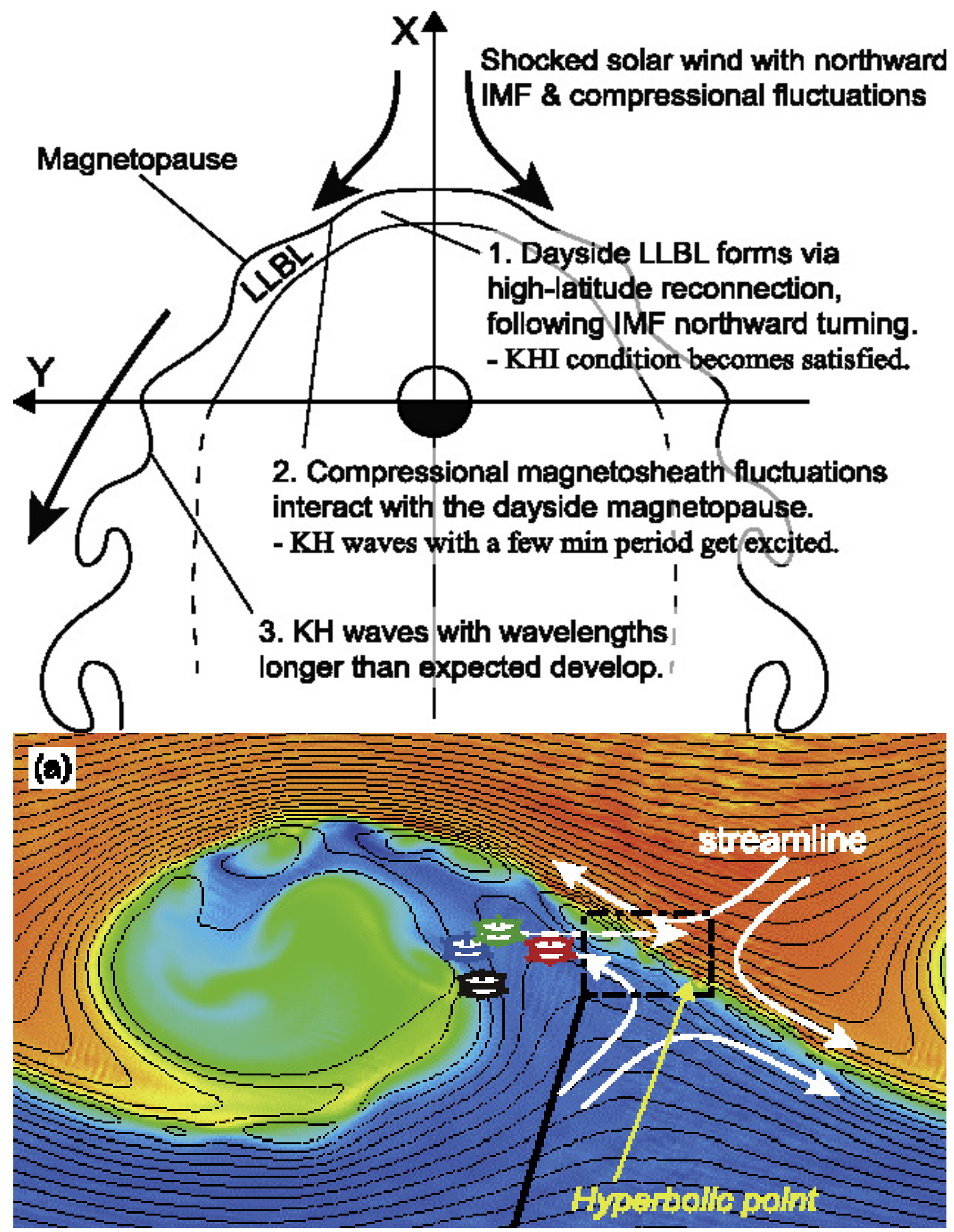

Figure 3: (top) Schematic of the equatorial magnetosphere showing how $\mathrm{KH}$ waves with wavelengths longer than predicted by the linear theory can be excited under northward IMF; (bottom) Two-dimensional two-fluid simulation of the KH instability [Nakamura et al., 2008], showing plasma density (red, dense; blue, tenuous) in a nonlinear stage, with in-plane magnetic field lines overlaid. The hyperbolic point is a stagnation point in the $\mathrm{KH}$-wave rest frame around which flow lines form hyperbolae and reconnection occurs (after Hasegawa (2009), copyright AGU). 

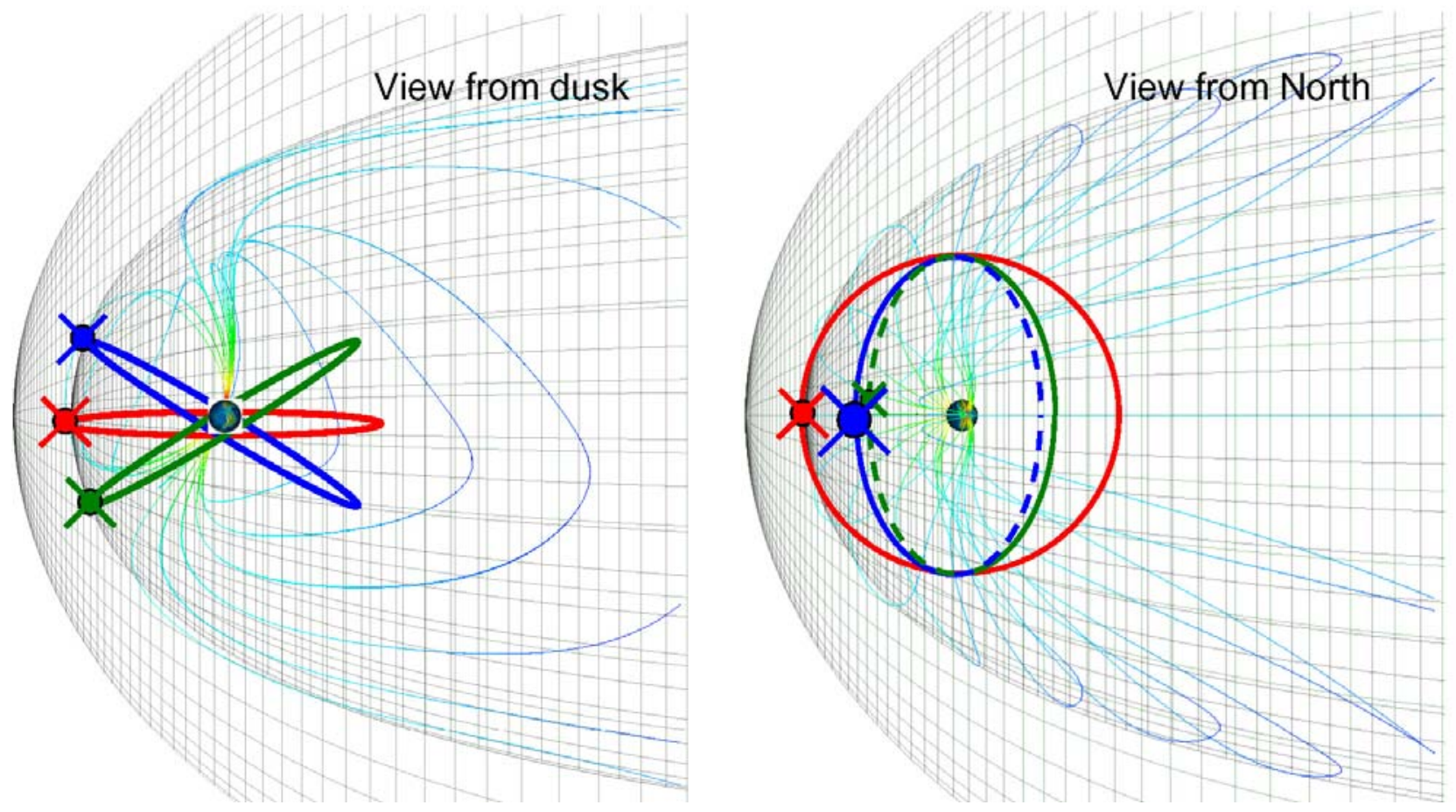

Figure 4: Schematic illustrations of the orbits of the 3 IMPALAS spacecraft. The left hand panel shows the view from dusk, with the 3 spacecraft located at the magnetopause boundary. The right hand panel shows the view from the north, and illustrates how the 3 spacecraft skim along the dayside magnetopause boundary for many hours during their 2-day orbit.

Figure 5: A cutaway of the design for dual head ion/electron spectrometer.

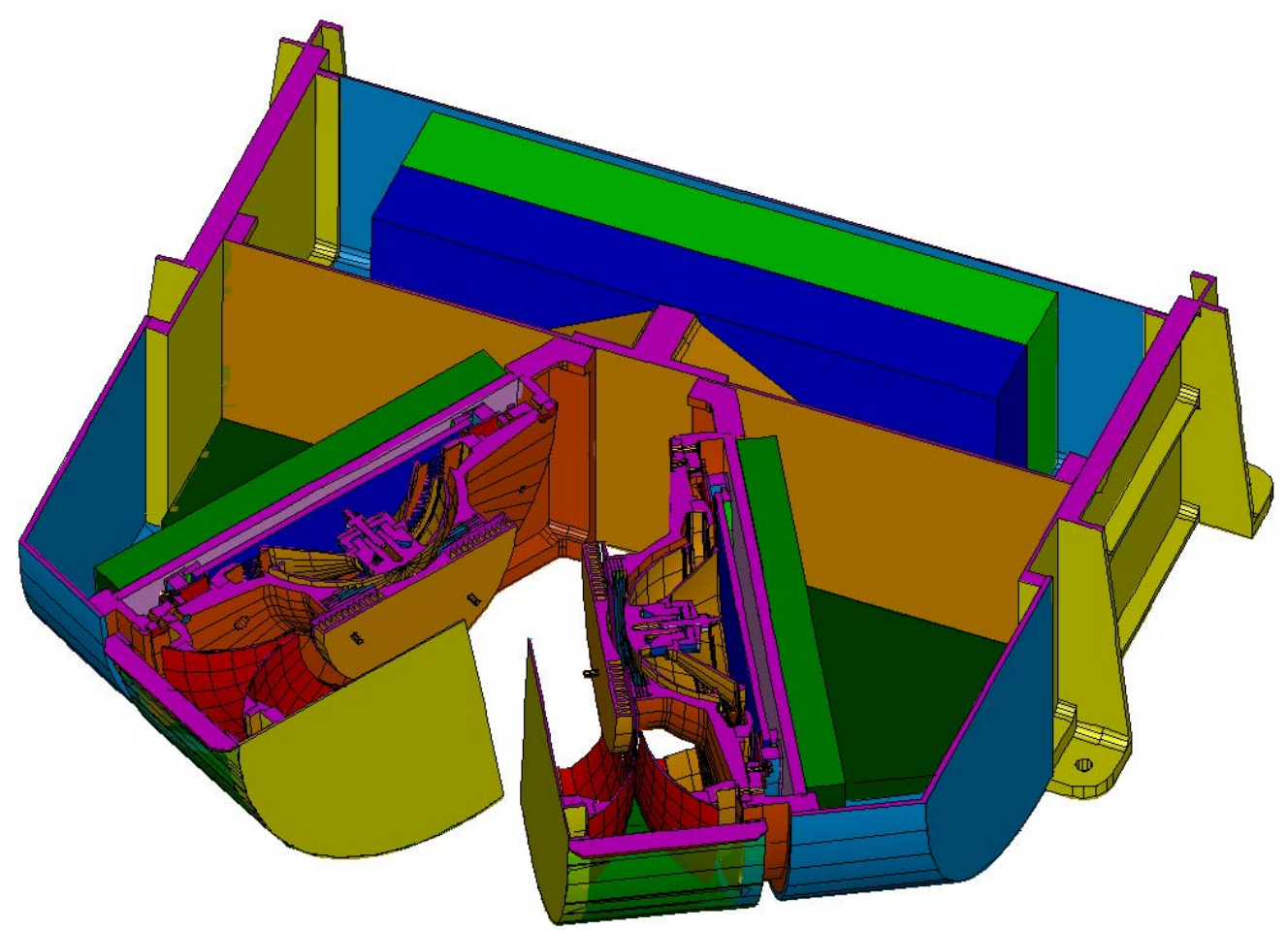




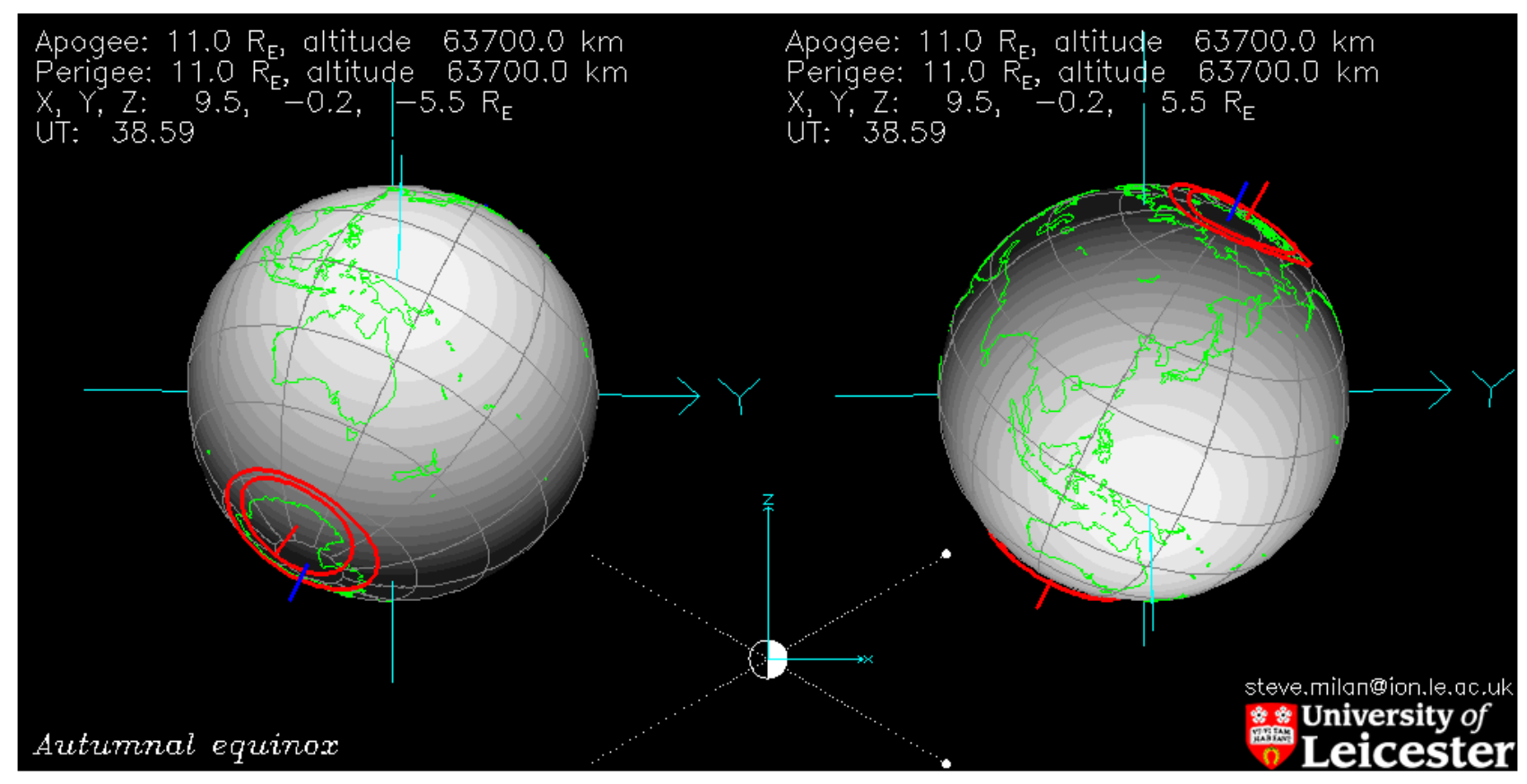

Figure 6. A snapshot from a simulation of the view of the dayside auroral oval (contained nominally within the red rings) obtained from the IMPALAS 2 and 3 spacecraft in inclined orbits. The simulation shows that the foot point of the field lines threading the 3 spacecraft would be visible in both northern and southern hemisphere.
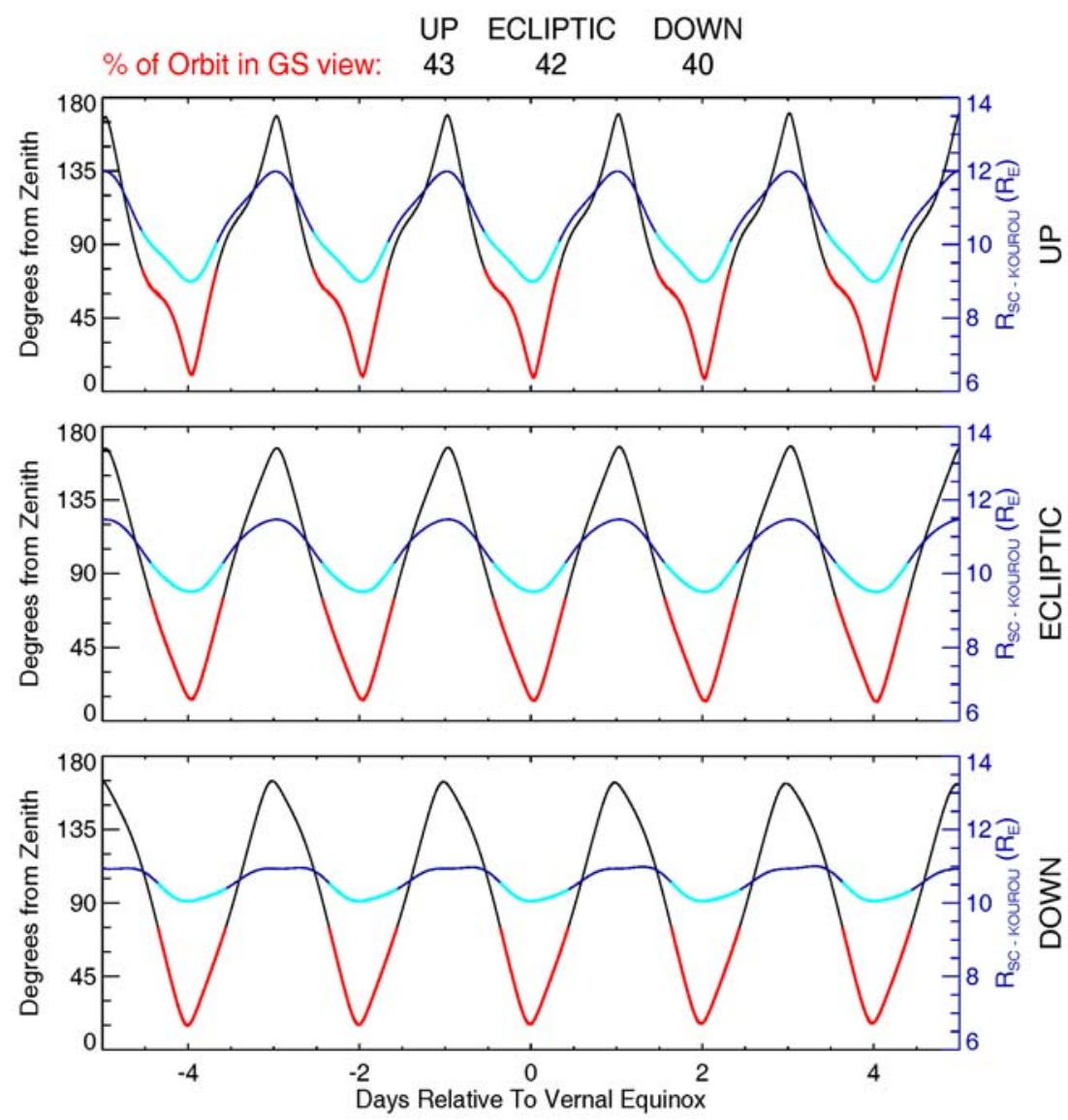

Figure 7: Coverage of the IMPALAS orbit with the Kourou ground station for a period of 10 days around spring equinox. Analysis shows there are $\sim 19$ hours per 48 hour orbit available for download (corresponding to red sections of plot when spacecraft are $>15$ deg above the horizon) when the spacecraft range from $9.0-10.5 R_{E}$ from the ground station. 